\title{
miR-125b can enhance skin tumor initiation and promote malignant progression by repressing differentiation and prolonging cell survival
}

\author{
Liang Zhang, Yejing Ge, and Elaine Fuchs \\ Howard Hughes Medical Institute, Laboratory of Mammalian Cell Biology and Development, The Rockefeller University,
} New York, New York 10065, USA

Previously, we identified $\mathrm{miR}-125 \mathrm{~b}$ as a key regulator of the undifferentiated state of hair follicle stem cells. Here, we show that in both mice and humans, miR-125b is abundantly expressed, particularly at early stages of malignant progression to squamous cell carcinoma (SCC), the second most prevalent cancer worldwide. Moreover, when elevated in normal murine epidermis, $m i R-125 b$ promotes tumor initiation and contributes to malignant progression. We further show that $\mathrm{miR}-125 b$ can confer "oncomiR addiction" in early stage malignant progenitors by delaying their differentiation and favoring an SCC cancer stem cell (CSC)-like transcriptional program. To understand how, we systematically identified and validated miR125b targets that are specifically associated with tumors that are dependent on $\mathrm{miR}-125 \mathrm{~b}$. Through molecular and genetic analysis of these targets, we uncovered new insights underlying miR-125b's oncogenic function. Specifically, we show that, on the one hand, mir-125b directly represses stress-responsive MAP kinase genes and associated signaling. On the other hand, it indirectly prolongs activated (phosphorylated) EGFR signaling by repressing Vps $4 b$ (vacuolar protein-sorting 4 homolog B), encoding a protein implicated in negatively regulating the endosomal sorting complexes that are necessary for the recycling of active EGFR. Together, these findings illuminate $\mathrm{miR}-125 b$ as an important microRNA regulator that is shared between normal skin progenitors and their early malignant counterparts.

[Keywords: squamous cell carcinoma; miR-125b; oncomiR; stress; stem cells; microRNA targets]

Supplemental material is available for this article.

Received July 1, 2014; revised version accepted October 3, 2014.

MicroRNAs (miRNAs or miRs) are a class of small regulatory RNAs that can have a major impact on the biology of both normal tissues and their associated cancers. In the mammalian genome, three distinct miRNAsmiR-99a/100, let-7a/c, and miR-125b-form an adjacently positioned and cotranscribed polycistronic miRNA cluster (Emmrich et al. 2014) whose alterations are frequently found in cancers (Calin et al. 2004). While let-7 family miRs are well-established suppressors (Johnson et al. 2005; Mayr et al. 2007), the roles of other individual members of this miR cluster in cancer are less clear.

In hematopoiesis, $m i R-125 b$ has been reported as an oncogene (Bousquet et al. 2008, 2010; Klusmann et al. 2010; Enomoto et al. 2011; Chaudhuri et al. 2012; Guo et al. 2012). However, evidence has favored the view that it acts as a tumor suppressor for cutaneous and head/neck squamous cell carcinoma (skin SCC and HNSCC) (Henson

Corresponding author: fuchs@rockefeller.edu or fuchslb@rockefeller.edu Article is online at http://www.genesdev.org/cgi/doi/10.1101/gad.248377.114. et al. 2009; Xu et al. 2012; Nakanishi et al. 2014). Indeed, overall levels of $m i R-125 b$ are reduced in many solid tumors, including not only skin SCCs and their closely related HNSCCs but also gliomas, melanomas, osteosarcomas, and endometrial tumors as well as cancers of the bladder, breast cancer, and ovarian epithelium. Moreover, $m i R-125 b$ overexpression in a number of cancer cell lines derived from such tumors has been found to suppress their proliferation, migration, invasion, and colony formation in vitro (for review, see Sun et al. 2013; Banzhaf-Strathmann and Edbauer 2014).

Although these data provide a compelling case for a tumor-suppressive function, several reasons suggest that they may not be unequivocal. First, due to the tightly

(C) 2014 Zhang et al. This article is distributed exclusively by Cold Spring Harbor Laboratory Press for the first six months after the full-issue publication date (see http://genesdev.cshlp.org/site/misc/terms.xhtml). After six months, it is available under a Creative Commons License (Attribution-NonCommercial 4.0 International), as described at http:// creativecommons.org/licenses/by-nc/4.0/. 
linked and polycistronically transcribed nature of the miR-99a/100-let-7a/c-miR-125b cluster, it is very difficult to separate the effects of these distinct miRNAs based on correlation analysis of either genomic alteration or expression change. Second, tumor-initiating, so-called cancer stem cells (CSCs) often represent only a small fraction of a solid tumor, and this could obscure the significance of measuring total $m i R$ levels within a heterogeneous tumor. Third, relationships between the expression and function of miRs are dependent on not only miR abundance but also the level of its target transcripts and the relative competition among these targets (Sumazin et al. 2011; Tay et al. 2011).Therefore, direct comparison of miR expression changes across markedly different cellular contexts (e.g., tumor vs. normal cells) may not accurately reflect corresponding changes in miR activity. Finally, whether tumorigenic or normal, stem cells are highly dependent on their specific niche environment, which may not be accurately recapitulated in vitro.

These issues highlight the importance of functionally testing the roles of $m i R-125 b$ in solid tumors in vivo. In this regard, several xenotransplantation experiments with miR-125b-overexpressing cell lines have been conducted, showing accelerated growth of xenotransplanted prostate, glioma, and breast tumor lines (Shi et al. 2011; Tang et al. 2012; Jin et al. 2013). Although the underlying mechanisms were not explored in detail in those studies, the results from those studies constitute the slim current evidence that miR-125b may function as an oncogene in these solid tumors.

Previously, we characterized miR-125b as a highly enriched miRNA in hair follicle (HF) stem cells (HFSCs) relative to their committed, proliferative, and short-lived progenies (Zhang et al. 2011). Moreover, when high $m i R$ $125 b$ expression was transgenically sustained in HFSCs, their early progeny in the outer root sheath (ORS) retained features of stemness at the expense of committed, differentiating progenies, thereby suggesting a role for miR-125b in normal skin stem cell maintenance (Zhang et al. 2011).

The importance of miR-125b in governing normal skin stem cells led us to wonder what its potential role might be in malignant transformation to SCCs, which are common skin cancers that originate from either HFSCs or basal epidermal progenitors and can metastasize and become life-threatening. Once formed, the growth of SCC is fueled by CSCs (Malanchi et al. 2008; Lapouge et al. 2011; White et al. 2011; Driessens et al. 2012) that share several key features of stemness: They can self-renew long-term and differentiate into lineage-restricted progenies of their tissuein this case, the SCC. At the transcriptional level, however, SCC-CSCs differ dramatically from normal skin stem cells, with $>700$ mRNAs changed by two times or more in CSCs relative to stem cells of either the epidermis or HF (Schober and Fuchs 2011; Lapouge et al. 2012).

In the present study, we used an in vivo genetic model to investigate the role of miR-125b in the initiation, progression, and maintenance of SCC in the native context of skin and in mice replete with their normal immune arsenal. We discovered that sustained miR-125b overexpression in this system promotes spontaneous tumor formation and also sensitizes skin to chemically induced carcinogenesis. Moreover, we found that at high levels, miR-125b can confer "oncomiR addiction" by repressing differentiation, a feature reminiscent of its role in normal stem cells and which can lead to a hyperproliferative state. Finally, by systematically analyzing the progressive changes in the gene expression profile that associate with $m i R-125 b$ oncomiR addiction and then carrying out functional studies, we uncovered a number of novel miR-125b target genes involved in growth factor and MAP kinase (MAPK) signaling pathways that are integral to its oncogenic function.

\section{Results}

miR-125b is an abundantly expressed miRNA in early stage tumorigenesis in skin

To investigate $m i R-125 b$ expression during the initiation and early progression of epithelial skin tumors, we conducted miRNA in situ hybridizations on normal adult mouse skin and on tumors induced by DMBA/TPA. In this carcinogenesis protocol, initial oncogenic mutations are introduced by a one-time topical application of mutagen DMBA. Benign papillomas then appear following multiple applications of the mitogen TPA. With additional TPA treatments, these tumors can progress to their more malignant SCC counterparts (Abel et al. 2009). Intriguingly, even though benign papillomas and their malignant SCC counterparts can derive from both epidermal stem cells and HFSCs (Malanchi et al. 2008; Lapouge et al. 2011; White et al. 2011; Driessens et al. 2012), miR-125b levels in SCC-CSCs were significantly higher than in basal epidermal cells but quite similar to HFSC levels (Fig. 1A). Moreover, miR-125b was not restricted to the tumor-stroma interface where CSCs reside but rather throughout the epithelial tumor tissue (Supplemental Fig. S1A).

Quantitative PCR (qPCR) analysis of normal skin stem cells and SCC-CSCs purified by fluorescence-activated cell sorting (FACS) (Schober and Fuchs 2011; Lapouge et al. 2012) revealed that miR-125b levels in SCC-CSCs were intermediate between epidermal basal progenitors and HFSCs isolated from the bulge niche (Fig. 1B; Schober and Fuchs 2011). However, miR-125b levels were very low in SCC-CSCs derived from repetitive DMBA treatment of mice whose skin was conditionally targeted for $T g f b r 2$, indicating variability at the malignant state.

Parallel to the above findings in mice, miR-125b expression in normal human skin was enriched in the bulge region of HFs compared with the epidermis. Additionally, elevated $m i R-125 b$ levels were found in lesional skin from actinic keratosis, which is preneoplastic growths that can progress to SCC (Supplemental Fig. S1B; Cockerell 2000). Finally, in comparison with 13 different control sections of normal human epidermis, 93 SCCs displayed considerable variation in in situ hybridization patterns of miR$125 b$ (Fig. 1C,D). Early stage (grade 1) SCCs frequently displayed abundant and widespread miR-125b signals that sometimes were higher than normal epidermis 
A

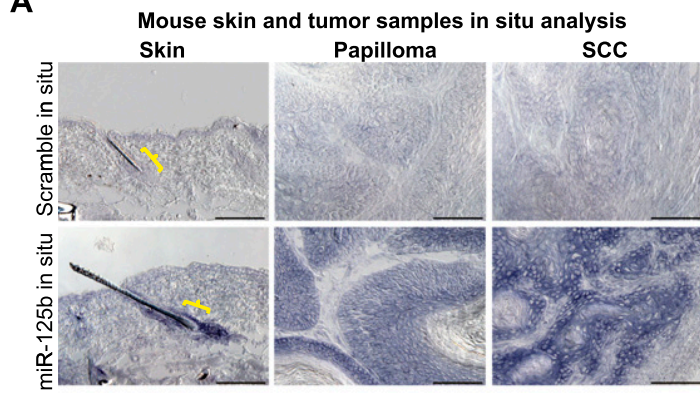

C

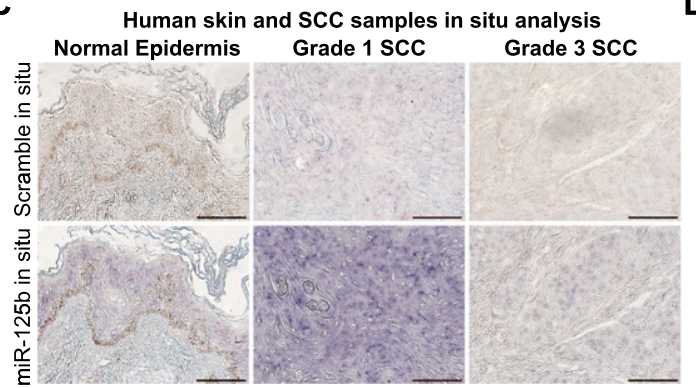

B
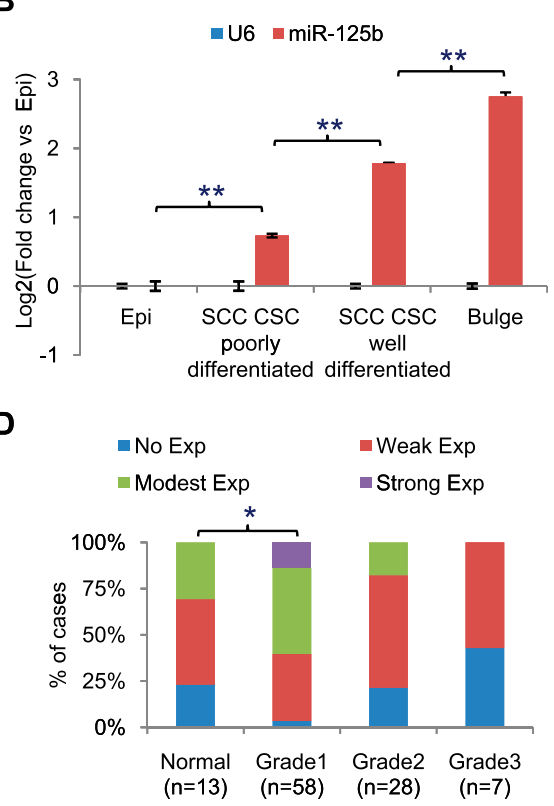

Figure 1. $m i R-125 b$ is an abundantly expressed miRNA in the early stages of skin tumorigenesis. $(A)$ In situ hybridizations of wildtype (WT) mouse skins whose HFs are in resting phase (telogen) and tumor tissues from DMBA/TPA-induced papillomas and SCCs. Bars, $100 \mu \mathrm{m}$. Yellow brackets indicate the bulge region, which harbors HFSCs. (B) qPCR analysis of miR-125b levels in SCC-CSCs and normal adult skin stem cells. (Bulge) Bulge HFSCs; (Epi) epidermal basal progenitors. CSCs $\left(\alpha 6^{\mathrm{Hi}} \beta 1^{\mathrm{Hi}}\right)$ were from DMBA-induced skin SCCs of wild-type mice (well differentiated) or mice conditionally ablated for TGF $\beta$ receptor II (poorly differentiated). $(C, D)$ In situ hybridizations were performed on a tissue array of human epidermis and different grade SCCs. Representative examples are in $C$. Bars, $100 \mu \mathrm{m}$. (D) Summary of miR-125b expression levels. The vertical axis indicates the percentage of tumors analyzed ( $n=$ number) that exhibited no, weak, modest, or strong expression. $\left(^{\star}\right) P<0.05, \chi^{2}$ test.

(Fig. 1C). In advanced (grade 3) SCCs, however, miR-125b signals were generally down-regulated.

Together, these findings highlighted parallels between mice and humans with regards to $m i R-125 b$ expression in both normal and tumorigenic skin. However, assessing the significance of these tumor progression-related changes in miR-125b expression necessitated functional studies in which miR-125b expression could be regulated independently of its tightly linked and polycistronically transcribed miRNA gene neighbors, which include the tumor suppressor let-7 (Supplemental Fig. S1C; Emmrich et al. 2014).

\section{miR-125b prolongs anchorage-independent survival of keratinocytes}

$m i R-125 b$ has been reported to repress in vitro proliferation and colony formation in a wide variety of both normal and tumor human cell lines, including those derived from human SCCs (Xu et al. 2012; Sun et al. 2013). We also observed that miR-125b overexpression reduces proliferation and colony formation of mouse keratinocytes (MKs) in vitro (Supplemental Fig. S2A; Zhang et al. 2011). In vivo, however, miR-125b overexpression led to the expansion of progenitor cells in both HFs and the epidermis (Zhang et al. 2011). Moreover and in contrast to normal epidermis, where proliferating progenitors are restricted to the single inner layer of integrinrich basal cells, proliferating cells extended into suprabasal layers analogous to that seen in premalignant and malignant skin tumors (Fig. 2A). The system that we used for these studies was our previously established K14-rtTA,TRE-miR$125 b$ double-transgenic (DTG) mouse, which allowed us to systemically deliver doxycycline (Dox) to induce miR-125b overexpression in skin epithelium (Zhang et al. 2011).

In vitro, the ability to survive and proliferate in suspension cultures can be likened to the absence of attachment to an underlying substratum in vivo and is a feature that commonly distinguishes malignantly transformed epithelial cells from their normal counterparts (Liotta and Kohn 2004). Interestingly, when placed in suspension, both normal MKs and bulge stem cells quickly lost viability (anoikis) under conditions where SCC-CSCs continued to proliferate (Supplemental Fig. S2B). The presence of suprabasal proliferation in DTG skin led us to wonder whether overexpressing miR-125b might also promote anchorage independence in MKs. Consistent with this hypothesis, in suspension culture, Dox-induced miR-125b overexpression prolonged survival under conditions where DTG MKs lost viability (Fig. 2B). These miR-125b-mediated effects appeared to be conserved in human keratinocytes, as judged by $m i R$ $125 b$ overexpression in HaCAT, an immortalized but otherwise normal human keratinocyte cell line (Fig. $2 \mathrm{C})$. These findings were particularly intriguing given that miR-125b overexpression in both MKs and human keratinocytes delayed growth in attached culture conditions (Supplemental Fig. S2C,D). 
A

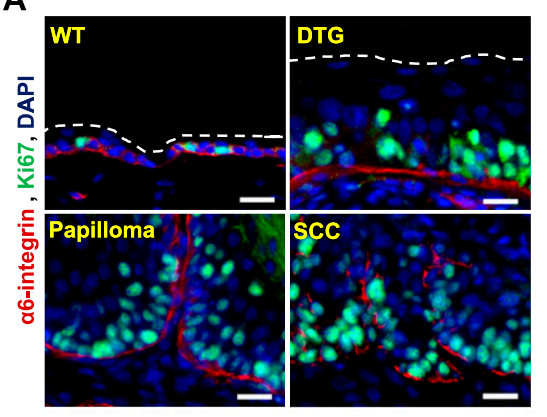

D

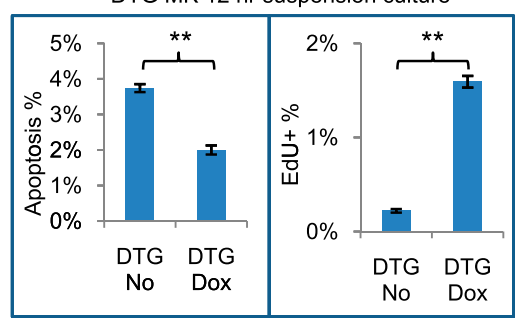

$\mathbf{F}$
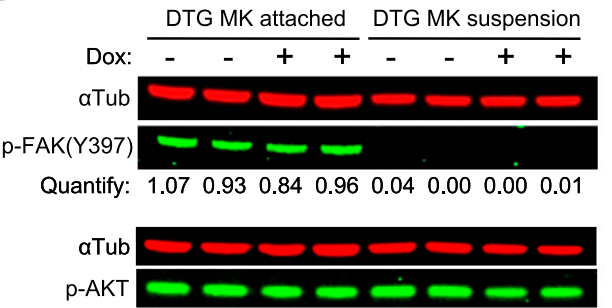

Quantify: $\begin{array}{llllllll}0.86 & 1.14 & 0.87 & 0.83 & 0.94 & 0.84 & 0.86 & 0.83\end{array}$
B

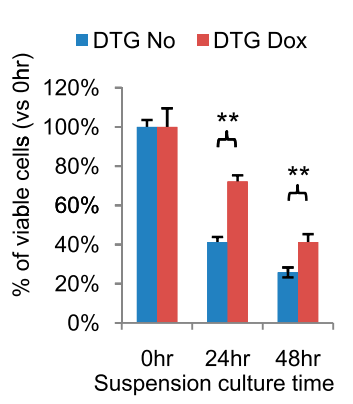

C

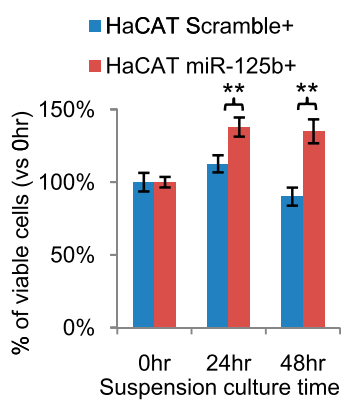

E HaCAT $12 \mathrm{hr}$ suspension culture

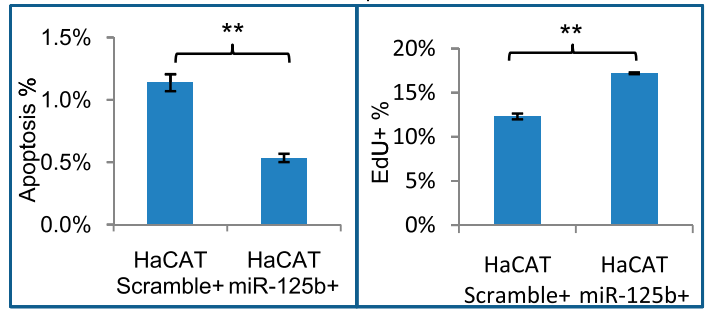

G

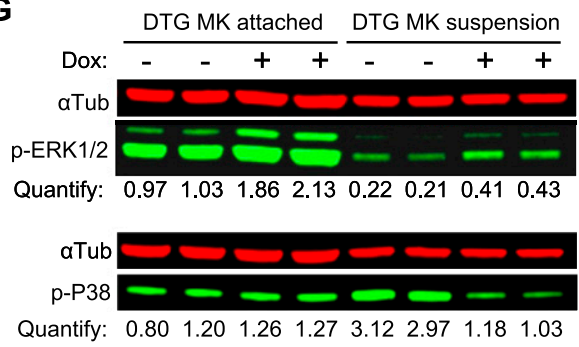

Figure 2. miR-125b promotes anchorage-independent survival. (A) Immunofluorescence of frozen skin sections from adult female mice. (Top row) Section at postnatal day 35 of wild-type (WT) and 3-wk Dox-induced DTG mice. (Bottom row) DMBA/TPA-induced wild-type mice displaying papilloma and SCC. The white line marks the skin surface. Bars, $20 \mu \mathrm{m}$. $(B, C)$ Cell proliferation/survival assays (MTT kit, Cell Biolabs, Inc.) of DTG MKs and HaCAT cells in suspension culture $\left(n=8\right.$ independent wells each). $\left(^{\star}\right) P<0.05$; $\left(^{\star \star}\right) P<0.01$, unpaired two-tailed $t$-test. $(D, E)$ The percentage of apoptotic/anoikis cells (AnnexinV [+] and dead cell stain [-]) and S-phase cells (EdU ${ }^{+}$after $1 \mathrm{~h}$ of labeling prior to harvesting) following $12 \mathrm{~h}$ in suspension culture of Dox-induced (Dox) or uninduced (No) DTG MKs $(D)$ or HaCAT-overexpressing $m i R-125 b$ or scrambled control $(E)(n=3$ independent wells). (F, G) Two-color quantitative immunoblot of DTG MK cells in attached culture and after $12 \mathrm{~h}$ in suspension culture. $(\alpha \mathrm{Tub}) \alpha$ Tubulin loading control; $(-)$ uninduced; (+) Dox-induced. Proteins of interest (green channel) signals were normalized to corresponding $\alpha$ Tubulin signals and compared with the average of uninduced DTG MKs in attached culture. Each lane is an independent well.

Using Annexin V staining to gauge apoptosis/anoikis and 5-ethynyl-2'-deoxyuridine (EdU) incorporation as a gauge for actively cycling cells, we found that in both MKs and human keratinocytes, $m i R-125 b$ overexpression improved both anchorage-independent survival and proliferation (Fig. 2D,E). Similar effects of $m i R-125 b$ overexpression were also observed for suspension, but not attached, cultures of human SCC cell lines from skin (A431) and the head and neck (SCC-15 and Fadu) (Supplemental Fig. S2E,F). These results were noteworthy in light of a recent report that $m i R-125 b$ protects mesenchymal stem cells from anoikis (Yu et al. 2012 ), suggesting that the role of $m i R-125 b$ in anchorageindependent survival may extend to multiple cell types.

Probing the mechanism, we found that similar to wildtype keratinocytes (i.e., uninduced DTG MK), Dox-induced miR-125b-overexpressing MKs still lost activated integrin signaling and hence activated (phosphorylated) focal adhesion kinase (FAK) when placed in suspension but showed no change in attached cultures. Activated (phosphorylated) AKT levels also appeared to be refractory to $m i R-125 b$ levels (Fig. 2F). In contrast, activation of ERK1/2 and P38 both showed sensitivity to $m i R-125 b$ levels in suspension, with ERK1/2 showing more activity and P38 showing less activity than their wild-type counterparts (Fig. 2G). Moreover, while a $m i R-125 b$-induced increase in ERK1/2 was also seen in attached cultures, the miR-125b-based reduction in P38 was not. Together, these findings expose an intriguing entanglement between $\mathrm{miR}-125 \mathrm{~b}$ and both growth factor-regulated and stress-regulated MAPK signaling pathways (see further analysis below).

\section{miR-125b acts as an oncomiR when overexpressed} in skin

The similarities in expression and in vitro behaviors of $m i R-125 b$ that we uncovered suggested that miR-125b's 
functions in keratinocytes may be conserved between mice and humans. To explore miR-125b's function in skin tumorigenesis, our DTG mouse model served as an ideal system, since it allowed us to regulate miR-125b levels independently of polycistronic transcripts from the endogenous miR-99a/100-let-7a/c-miR-125b cluster. Interestingly, DTG mice derived from three independently generated TRE-miR-125b transgenic lines consistently developed spontaneous skin tumors after sustained Dox induction (Fig. 3A; Supplemental Fig. S3A). Spontaneous tumor formation was highly penetrant, and, by $5 \mathrm{mo},>90 \%$ of miR-125b-overexpressing mice examined had developed at least one tumor (all 13 on-Dox DTG mice used to collect the data in Fig. 3A developed skin tumors before death in the experiment's time frame). In contrast, Dox-treated wild-type littermates and uninduced DTG mice remained

A

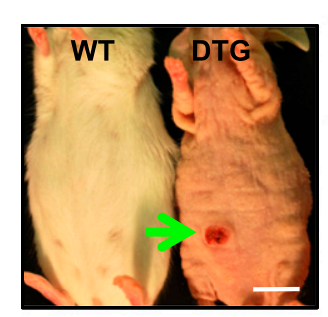

F9 line, Dox 76d

B

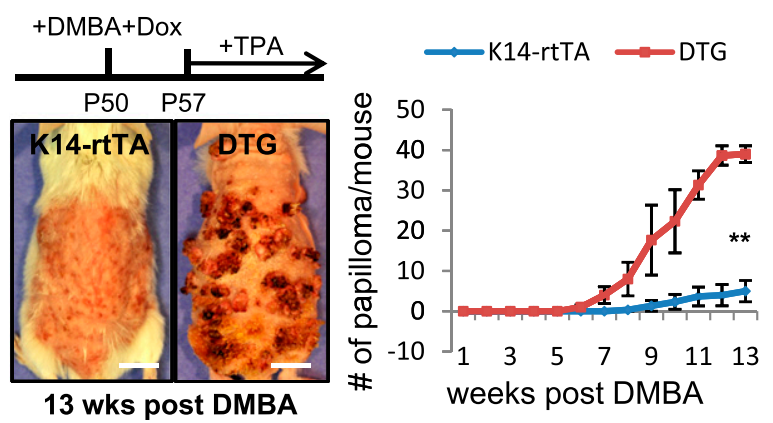

C

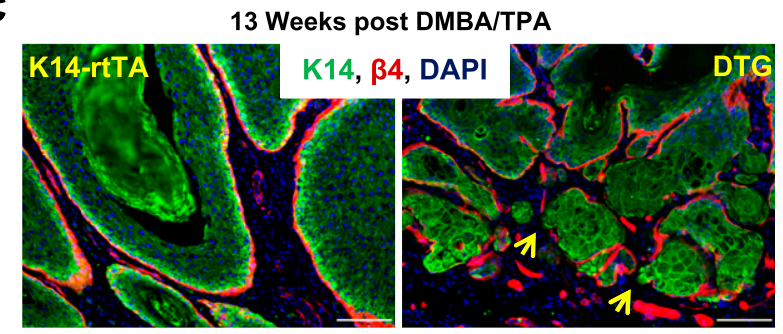

D Serial transplanted (passage 5)DMBA/TPA induced DTG tumor

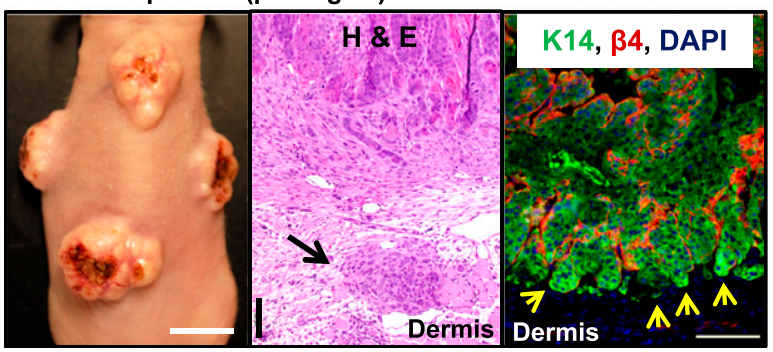

tumor-free during this time (Fig. 3A). These tumors persisted in the presence of Dox but regressed quickly upon Dox withdrawal, indicating their dependence on miR-125b (Supplemental Fig. S3B).

We also tested the sensitivity of DTG mice to DMBA/ TPA chemical carcinogenesis. To minimize secondary effects that might arise from miR-125b-induced skin hyperplasia and loss of differentiation, we administered Dox simultaneously with the DMBA mutagen. Strikingly, both the speed and frequency of tumorigenesis were greatly enhanced in DTG mice relative to singletransgenic controls or uninduced DTG controls (Fig. 3B; Supplemental Fig. S3C). Interestingly, TPA alone, but not DMBA alone, induced papilloma formation on DTG skin, although at a much lower frequency than DMBA/TPA treatment (Supplemental Fig. S3D).

As time progressed, miR-125b overexpression resulted in DMBA/TPA-induced tumors that displayed irregular borders and regions of discontinuity in the basement membrane, which are early hallmarks of malignant progression (Fig. 3C). In contrast, K14-rtTA control mice treated in parallel generated only benign papillomas during the time span of the regimen. Together, these results suggested that miR-125b overexpression alone functions as a weak tumor initiator that can be further enhanced by additional mutations caused by DMBA.

Malignant progression to SCC normally requires more than at least 5 mo of DMBA/TPA (Hennings et al. 1983), but Dox treatment of DTG mice had to be discontinued after 3 mo due to rapid tumor progression. To test for the presence of SCC-initiating cells within tumors, we therefore turned

Figure 3. $m i R-125 b$ overexpression is oncogenic in skin. $(A)$ Spontaneous tumor formation observed in skins from $m i R$ 125b-overexpressing DTG transgenic mice (F9 line) but not control (wild-type [WT]) FVB female mice (five different litters). Accompanying Kaplan-Meier curves show tumor-free survival rates. Mice were Dox-induced (or not) and tracked since 3 wk of age. $(B)$ Experimental approach for DMBA/TPA-induced carcinogenesis and representative DTG mouse and control littermates after 13 wk of DMBA/TPA treatment. All mice were on Dox for the duration of the experiment. The accompanying graph plots the average tumor number per mouse at the times indicated. All mice were FVB background age-matched females $(n=3$ for each curve; error bars represent standard error). $(C)$ Immunofluorescence of sections of 13-wk tumors induced with (DTG) or without (K14-rtTA) miR-125b overexpression. Arrows denote sites of disrupted basement membranes at the tumorstroma interface, a hallmark of SCC. Similar features were observed on more than five independent tumors. $(D$, left $)$ Tumors formed from $\alpha 6^{\text {hi }} \beta 1^{\text {hi }}$ DTG cells serially transplanted five times into mice fed on Dox food. Tumors from $1 \times 10^{4}$ engrafted cells per site were allowed to grow for $1 \mathrm{mo}$. Similar results were independently reproduced more than three times. (Middle) Histology of a representative engrafted DTG tumor. Arrows indicate invasive tumor mass within the dermis. Similar malignancies were observed with six independently engrafted tumors. (Right) Immunofluorescence of a section from a representative engrafted DTG tumor. Similar features were observed on three independently engrafted tumors. Bars: left panel, $1 \mathrm{~cm}$; other panels, $100 \mu \mathrm{m}$. 
to FACS to purify and characterize their integrin $\alpha 6^{\mathrm{Hi}} \beta 1^{\mathrm{Hi}}$ epithelial cells. Similar to $\alpha 6^{\mathrm{Hi}} \beta 1^{\mathrm{Hi}}$ stem cells from normal HFs and SCCs (Blanpain et al. 2004; Schober and Fuchs 2011), these $\alpha 6^{\mathrm{Hi}} \beta 1^{\mathrm{Hi}}$ tumor cells readily formed large colonies or holoclones ( $>20$ cells per colony) in attached cultures (without Dox) in vitro (Supplemental Fig. S3E).

Moreover and consistent with previously established features of SCC-CSCs (Schober and Fuchs 2011), $\alpha 6^{\mathrm{Hi}} \beta 1^{\mathrm{Hi}}$ DTG tumor cells initiated secondary tumor growth with higher efficiency than $\alpha 6^{\text {lo }} \beta 1^{\text {lo }}$ counterparts when intradermally transplanted directly (without culture) into the backskins of immunocompromised (Nude) mice (Supplemental Fig. S3F). Additionally, when $\alpha 6^{\mathrm{Hi}} \beta 1^{\mathrm{Hi}}$ cells were FACs-purified directly from these secondary tumors and serially transplanted, they continued to initiate tumors, which were judged by histopathological analysis to be invasive SCCs (Fig. 3D). Persistent miR-125b overexpression in these serially transplanted tumor cells was confirmed by qPCR (Supplemental Fig. S3G), providing compelling evidence that $\mathrm{miR}$-125b-overexpressing tumors contained serially transplantable tumor-initiating CSCs (referred to here as DTG-CSCs). Finally, consistent with our prior finding that $m i R-125 b$ overexpression stimulates sebaceous gland growth (Zhang et al. 2011), Oil-Red-O-positive sebocytes were found in tumors from Dox-induced DTG skin whether spontaneous, DMBA/TPA-stimulated, or serially transplanted (Supplemental Fig. $\mathrm{S} 3 \mathrm{H}$ ).

\section{$D M B A / T P A$-induced SCC tumors that arise in DTG mice are addicted to $\mathrm{miR}-125 \mathrm{~b}$}

Some oncogenes function in not only initial development of a specific tumor but also maintaining its malignant state, known as oncogene addiction (Weinstein 2002). To determine whether $m i R-125 b$ has this capacity, FACSpurified DTG-CSCs were transduced with a lentivirus expressing fluorescent histone H2BGFP and then directly engrafted into Dox-fed Nude mice to induce miR-125bmediated tumor growth. When tumors appeared, half of the mice were switched to regular food (off-Dox). While on-Dox tumors kept growing, off-Dox tumors regressed rapidly (Fig. 4A; Supplemental Fig. S4A).

Immunolabeling of epithelia with keratin (K14) and nuclei (DAPI) revealed signs of tumor shrinkage within $4 \mathrm{~d}$ off-Dox; by $7 \mathrm{~d}$, cyst-like structures were seen (Fig. 4B, top panels). Correspondingly, the mitotic marker (phosphohistone H3) waned, while the epidermal differentiation marker (K10) rose (Fig. 4B). The rapid decline in tumor proliferation after Dox withdrawal was confirmed by EdU labeling and FACS analysis (Fig. 4C). Apoptotic markers, such as activated caspase 3 and TUNEL (to measure DNA fragmentation), displayed no discernable increase at $4 \mathrm{~d}$ off-Dox and only a slight increase at $7 \mathrm{~d}$. In contrast, the percentage of differentiated tumor cells $\left(\alpha 6^{\text {low }} \beta 1^{\text {low }}\right)$ in the FACS populations rapidly increased (Supplemental Fig. S4B,C).

To characterize the gene expression changes underlying these phenomena, we FACS-purified GFP $+\alpha 6^{\mathrm{Hi}} \beta 1^{\mathrm{Hi}}$ tumorinitiating cells at $t=0,4$, and $7 \mathrm{~d}$ off-Dox. qPCR of their RNAs confirmed the gradual decline of $m i R-125 b$ in regressing DTG tumors (Supplemental Fig. S4D). Illumina sequencing on these RNAs showed that progressive transcriptional changes accompanied $m i R-125 b$ withdrawal (Fig. 4D). During tumor regression, mRNAs encoding proliferation-related proteins (Ki67 and Cyclin D1) and SCCenriched keratins (K18 and K6) were down-regulated, while those encoding epidermal differentiation markers (K1, K10, and Loricrin) were up-regulated. These changes were consistent with the end of proliferation and rise of differentiation observed when $m i R-125 b$ was restored to its endogenous levels within these tumor masses.

\section{miR-125b maintains the molecular identity} of miR-125b-addicted SCC-CSCs

Unsupervised hierarchical analysis showed that initial (off-Dox 0 d) DTG-CSCs were transcriptionally similar to previously characterized profiles of SCC-CSCs (Schober and Fuchs 2011). Strikingly, as time progressed, this profile shifted to one that more closely resembled that of normal skin stem cells (Fig. 4E). To further quantify this shift, we first calculated the miR-125b correlation score (CS) for each protein-coding gene in the $m i R-125 b$ addicted tumor regression process: An mRNA whose expression is concomitantly decreased during temporal $m i R-125 b$ reduction will have a positive CS value, while one whose expression is increased will have a negative CS value. Based on these values, we conducted a gene set enrichment analysis (GSEA) (Mootha et al. 2003; Subramanian et al. 2005) on previously identified SCCCSC signature genes that were two times or more similar to each other than to stem cells of either epidermis or HFs (Schober and Fuchs 2011).

Consistent with our observation in Figure 4E, the SCCCSC up-regulated signature genes were significantly enriched for genes whose expression positively correlated with miR-125b level during DTG tumor regression. On the other hand, the SCC-CSC down-regulated signature genes were significantly enriched for genes whose expression negatively correlated with the miR-125b level during DTG tumor regression (Fig. 4F). In addition, a strong negative correlation with $m i R-125 b$ during tumor regression was found for genes that were previously identified to be down-regulated upon $m i R-125 b$ overexpression in normal HFSCs (Fig. 4G; Zhang et al. 2011). These data suggest that a significant proportion of putative $m i R-125 b$ target genes is shared between normal skin and tumor stem cells.

To test the general importance of $m i R-125 b$ for tumor stem cell activity, we attempted to knock down this miRNA in CSCs from SCCs in which miR-125b expression was controlled by its natural locus. We first constructed a miRNA "sponge inhibitor" for miR-125b in which multimerized $m i R-125 b$-binding sites were introduced into the $3^{\prime}$ untranslated region (UTR) of a GFP reporter (Ebert et al. 2007). After validating its functionality in cultured MKs, we then lentivirally transduced the GFP-miR-125b sponge and GFP-control into CSC line 63A, derived from SCCs that were generated by DMBA carcinogenesis applied to a wild-type (mostly C57B16) 
A

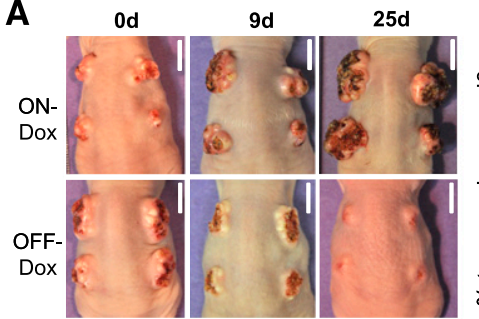

B

B DTG tumor Off-Dox regression (0d-7d)

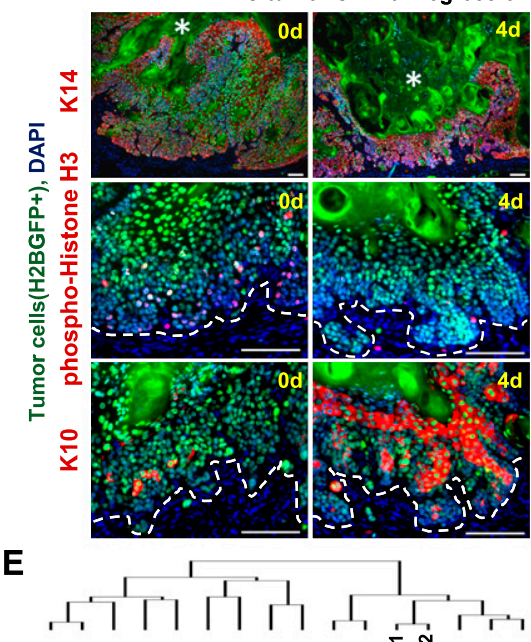

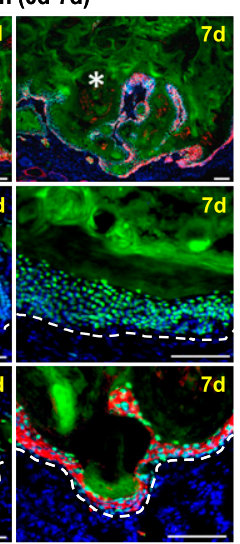

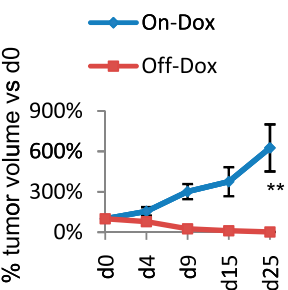

C

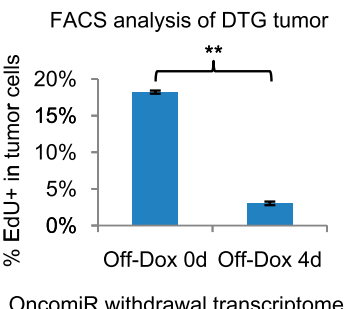

D OncomiR withdrawal transcriptome

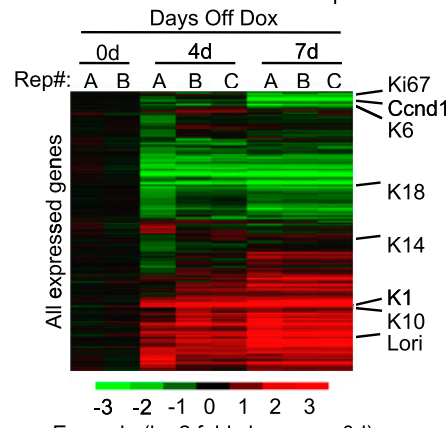

Example ( $\log 2$ fold change vs $0 \mathrm{~d}$ )

G miR-125b downregulated genes in normal skin SCs

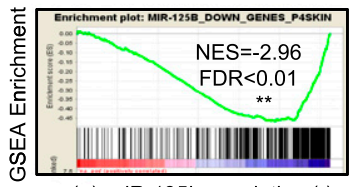

$(+)$ miR-125b correlation (-)

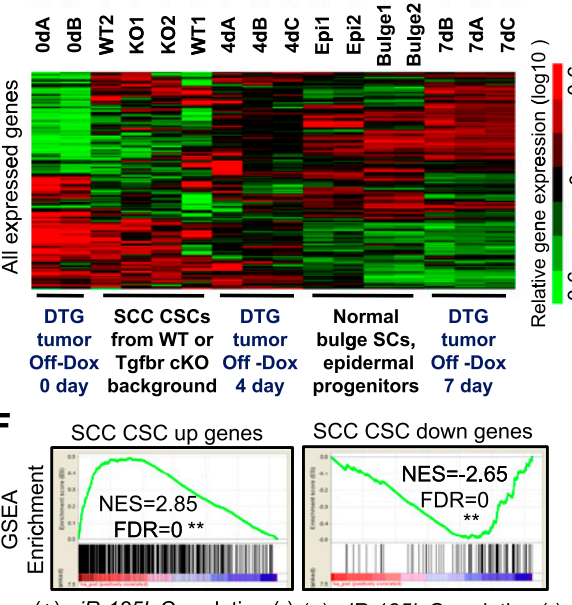

$(+) m i R-125 b$ Correlation (-) (+)miR-125b Correlation (-)

H Mouse SCC CSC serial dilution
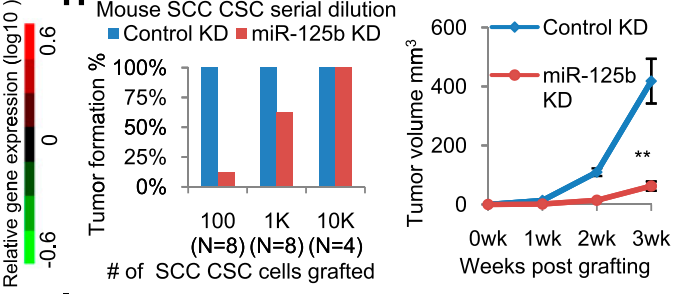

I
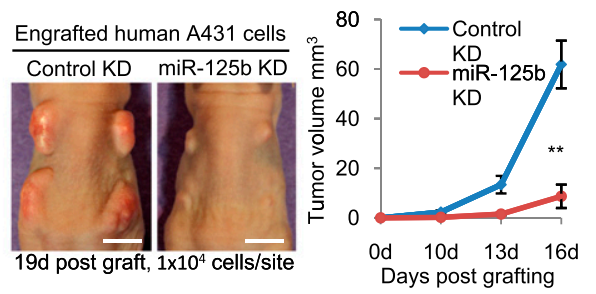

Figure 4. $m i R-125 b$ confers oncomiR addiction and maintains the molecular identity of DTG-CSCs. $(A)$ Nude mice were engrafted with $1 \times$ $10^{4} \mathrm{miR}-125 \mathrm{~b}$-overexpressing DTG-CSC cells per site and maintained on Dox until tumors grew to $\sim 1 \mathrm{~cm}$. Half the mice were then taken off Dox. Shown are representative mice from each cohort that were imaged at the times indicated. The graph shows quantification of tumor volume (percentage relative to $t=0)$ over time $\left(n=4\right.$ each). Error bars indicate standard error. $\left({ }^{\star}\right) P<0.01$ based on unpaired two-tailed $t$-test. $(B)$ Immunofluorescence of sections of regressing GFP+DTG tumors after Dox withdrawal (Off-Dox). Similar results were reproduced on three independently engrafted tumors each. Bars, $100 \mu \mathrm{m}$. ( ${ }^{\star}$ ) Cell-less cysts; (white line) tumor-stroma interface. (C) FACS analysis of live epithelial tumor cells from an off-Dox GFP+DTG tumor after $4 \mathrm{~h}$ of in vivo EdU labeling. $(D)$ Unsupervised hierarchical clustering (heat map) of the gene expression profile of GFP $+\alpha 6^{\text {hi }} \beta 1^{\text {hi }}$ cells (DTG-CSCs) from regressing GFP+DTG tumors. (Red) Up-regulation; (green) down-regulation. (E) Unsupervised hierarchical clustering (heat map) of the above regressing DTG-CSC transcriptional profile data sets with previously characterized transcriptional profile data sets of SCC-CSCs and normal skin stem cells (Schober and Fuchs 2011). (Bulge) Bulge HFSCs; (Epi) epidermal basal progenitors; (wild-type [WT] and knockout [KO]) SCC-CSCs (CD34 $\alpha 6^{\mathrm{Hi}} \beta 1^{\mathrm{Hi}}$ ) of DMBA-induced SCCs from wild-type skin or K14-Cre $\times$ TGF $\beta$ receptor II conditional knockout $(\mathrm{cKO})$ skin. To rule out platform bias, gene expression data were log-transformed, row-centered, row-normalized within each data set using Gene Cluster 3.0 software before combining the two data sets for clustering. $(F)$ Gene set enrichment analysis (GSEA) of the previously identified SCC-CSC up-regulated (left panels) or down-regulated (right panels) signature genes (two times or more vs. normal HFSCs) compared with the above regressing DTG-CSC gene expression data sets. Comparisons are against all of the expressed genes ranked by their miR-125b correlation score (CS) (see the text). (Horizontal axis) CS; (vertical axis) GSEA enrichment score; (NES) normalized enrichment score; (FDR) false discovery rate-adjusted $P$-value of enrichment. $\left.{ }^{\star \star}\right)$ FDR $<0.01$. $(G)$ GSEA analysis of mRNAs down-regulated in normal HFSCs after miR-125b overexpression (Zhang et al. 2011) compared with the above regressing DTG-CSC mRNA data set. All features are the same as above. $(H, l e f t)$ Tumor-forming efficiency of serially diluted SCC-63A cells after in vitro modification by miR-125b knockdown (KD) (miR-125b sponge + miR-125b antagomir) or control knockdown (control sponge + control antagomir). (Right) Growth curve of engrafted miR-125b knockdown or control knockdown SCC-63A tumors. $n=2$ mice with four tumors each. ( $I$, left) Tumors generated from A431 cells after in vitro modification by miR-125b knockdown (miR-125b antagomir) or control knockdown (control antagomir). (Right) Growth curve of engrafted miR-125b knockdown or control knockdown A431 tumors. $n=2$ mice with four tumors each. $\left(^{\star \star}\right) P<0.01$, repeated-measure ANOVA test (GraphPad Prism). Error bars indicate standard error (same for $H$ ). 
mouse (Schober and Fuchs 2011). To achieve optimal miR-125b knockdown, sponge-transduced cells were further transfected with $m i R-125 b$ (or scramble) antagomirs. $\mathrm{GFP}^{\mathrm{Hi}}$ cells were then FACS-purified and tested by qPCR to confirm miR-125b knockdown (Supplemental Fig. S4E-G). Relative to controls, miR-125b knockdown did not affect their in vitro proliferation and/or viability; however when miR-125b knockdown CSCs were injected into Nude mice, tumor initiation efficiency and growth rates were markedly diminished (Fig. $4 \mathrm{H}$; Supplemental Fig. S4H,I). Similar effects were seen with A431 cells, where miR-125b is expressed but at much lower levels than normal human skin (Supplemental Fig. S4J,K; Xu et al. 2012; Sun et al. 2013). Treatment of miR-125b antagomir significantly reduced their tumor growth rate upon their xenotransplantation into Nude mice (Fig. 4I).

\section{miR-125b is a positive regulator of EGFR pathway} activity

We next focused on putative targets of miR-125b that might contribute to its effects on skin tumorigenesis. One candidate was the tumor suppressor gene Trp53, which is a direct miR-125b target in some cells (Le et al. 2009). However, in our regressing skin tumors, both Trp53 and its target genes (Beronja et al. 2010) were down-regulated, not up-regulated, as miR-125b levels diminished (Supplemental Fig. S5A). Vdr was more promising in that this miR-125b target had been implicated in normal HFSCs (Zhang et al. 2011). In regressing DTG tumors, both $V d r$ and predicted VDR target genes rose in expression as $m i R-125 b$ levels waned (Supplemental Fig. S5B). However, when we administered Vdr ligand EB1089 (Palmer et al. 2008) under conditions known to partially suppress the effects of miR-125b overexpression in normal HFSCs (Zhang et al. 2011), we did not detect antagonistic effects in DTG-CSCs (Supplemental Fig. S5C).

We next turned to the EGFR-Ras pathway featured prominently in skin tumorigenesis. Although DMBA treatment often results in HRas1 mutations (Quintanilla et al. 1986), this is not always the case, particularly when there are other oncogenic mutations (Mao et al. 2004). Sequence analysis of the DTG-CSC line used to screen our miR-125b targets did not reveal mutations in the commonly hit HRas1 codon 61 or elsewhere in the HRas1 coding sequence (Supplemental Fig. S5D; data not shown).

We then applied the GSEA algorithm to analyze the correlation between miR-125b and EGFR-Ras-responsive genes during DTG tumor regression. A strong positive correlation was detected between miR-125b and (1) genes up-regulated upon EGF treatment of MCF-7 mammary carcinoma cells (MSigDB and M2634) (Creighton et al. 2006), (2) the 300 most significantly up-regulated genes in oncogenic KRAS immortalized human lung epithelial cells (AALE; MSigDB and M2882) (Barbie et al. 2009), and (3) genes whose promoters/enhancers harbor predicted binding motifs for ELK1 (MSigDB and M17817), a transcription factor downstream from EGFR-Ras signaling (Fig. 5A). In vitro, miR-125b overexpression in DTG MKs enhanced activation (phosphorylation) of ERK2, a downstream effector of EGFR-Ras signaling, while miR-125b knockdown in SCC-63A cells reduced ERK2 phosphorylation (Fig. 5B).

The lack of suitable antibodies for mouse phosphoEGFR led us to human HaCAT cells, where ERK2 was similarly hyperactivated upon miR-125b overexpression (Fig. 5C, left panel). miR-125b overexpression analogously elevated EGFR phosphorylation both in regular cultures (Fig. 5C, right panel) and upon EGF ligand stimulation of serum-starved cultures (Supplemental Fig. S5E). Finally, although ERK phosphorylation was suppressed by miR$125 b$ in Fadu cells (Nakanishi et al. 2014), EGFR phosphorylation was enhanced (Supplemental Fig. S5F).

Even though downstream effectors varied with cell type and environmental conditions, EGFR signaling positively correlated with miR-125b. Thus, we treated Nude mice bearing engrafted miR-125b-addicted DTG skin tumors with erlotinib, a small molecule inhibitor of EGFR activity (Raymond et al. 2000). Consistent with this link, erlotinib reduced the mitotic index, induced differentiation marker K10, and reduced overall tumor growth rate, partially mimicking the effects of miR-125b withdrawal in vivo (Fig. 5D,E).

miR-125b modulates EGFR activity by targeting Vps4b (vacuolar protein-sorting 4 homolog B)

miRNAs typically repress the levels and translation of their direct target mRNAs. Consistently, transcripts harboring a predicted seed sequence for miR-125b (but not another miRNA, Let-7) displayed a strong negative correlation with $m i R-125 b$ in our DTG tumor regression data set (Supplemental Fig. S5G). Positing that miR-125b may target a negative regulator of EGFR, we surmised that potential candidates should meet the following criteria: (1) Their expression should negatively correlate with miR$125 b$ during DTG tumor regression upon Dox withdrawal (CS $<-1.3$; i.e., negative correlation $P<0.05$ ), and $(2)$ their 3' UTRs should harbor conserved miR-125b-binding sites as predicted by TargetScan (Friedman et al. 2009).

One-hundred-sixty-five such putative miR-125b targets were identified (Supplemental Table S1). Particularly intriguing was $V p s 4 b$, encoding vacuolar protein-sorting 4 homolog B. Although VPS4B's function in SCCs is unexplored, in cultured breast cancer cells, it promotes endocytic degradation of phosphorylated EGFR and inhibits anchorage-independent growth (Lin et al. 2012). Consistently, VPS4B knockdown in cultured human SCC cells caused significant up-regulation of EGFR phosphorylation. Moreover, up-regulation of both $V p s 4 b$ mRNA and protein occurred concomitantly with miR-125b down-regulation during DTG tumor regression (Supplemental Table S1; Supplemental Fig. S5H,I). miR-125b overexpression also modestly affected VPS4B protein levels in both MKs and human keratinocytes and multiple human SCC lines (Fig. 6A; Supplemental Fig. S5J).

A conserved putative miR-125b-binding site exists in the 3' UTR of Vps $4 b$ mRNA (Supplemental Fig. S5K). To 
A

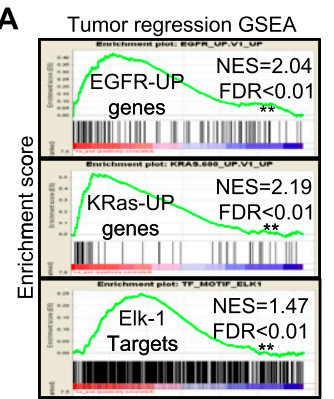

(+) miR-125b Correlation (-)

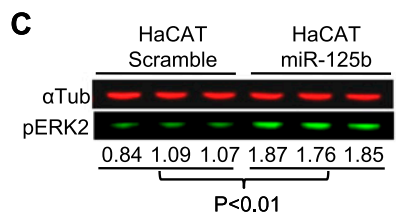

D Tumor cells (Histone H2BGFP+)

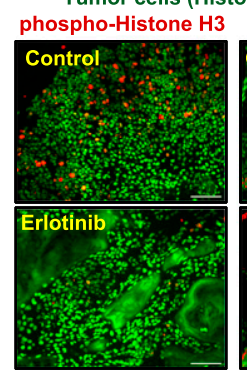

E

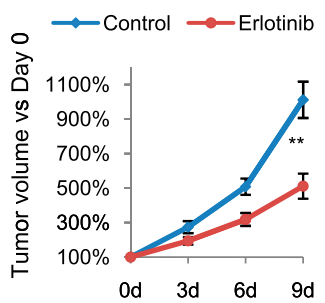

B
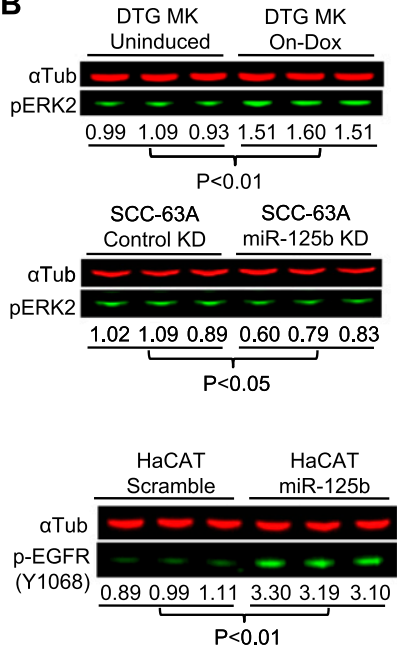

K10

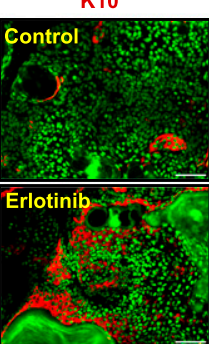

$\mathrm{PH} 3+\mathrm{K} 10+$
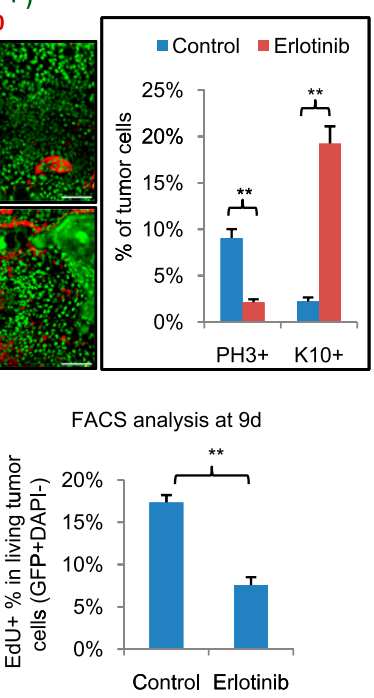

Figure 5. $m i R-125 b$ promotes EGFR pathway activity. (A) GSEA of previously identified EGFR pathway-responsive gene sets (from MSigDB; see the text for details) compared with the gene expression data of regression DTG-CSCs described above. All features are the same as Figure 4F. $(B, C)$ Two-color quantitative immunoblot analysis (each lane represents an independent well). ( $B$, top $)$ Cultured DTG MK cells \pm Dox induction; (bottom) SCC-63A cells harboring miR-125b knockdown (KD) (miR-125b sponge $+m i R-125 b$ antagomir) or control knockdown (control sponge + scrambled antagomir). (C) HaCAT cells overexpressing miR-125b or scrambled control. Protein of interest levels (green) are quantified relative to $\alpha$-tubulin loading controls (red) and compared with the average of the left three columns of each figure. $P$-values based on two-tailed $t$-test. $(D)$ Immunofluorescence of sections from engrafted H2BGFP-labeled DTG tumors after $3 \mathrm{~d}$ or erlotinib or solvent control treatment. The right panel shows quantification of the percentage of phospho-histone $\mathrm{H}^{+}\left(\mathrm{PH}^{+}\right)$mitotic cells and $\mathrm{K}^{+} 0^{+}$differentiating cells among all $\mathrm{GFP}^{+}$tumor cells. $n=3$ independent tumors. $\left(^{\star \star}\right)$ $P<0.01$ by two-tailed $t$-test. Error bars indicate standard error. (E, left panel) Growth curve of GFP+DTG tumors treated daily with erlotinib or solvent control. (Right panel) FACS analysis of these tumors at day 9 after $4 \mathrm{~h}$ of EdU labeling. $n=4$ independent tumors.

test its functionality, we constructed a reporter by linking luciferase cDNA to the full-length mouse $V p s 4 b$ 3' UTR. Reporter expression was significantly repressed by $m i R$ $125 \mathrm{~b}$ in cultured wild-type MKs, and this was abolished when the site was mutated (Fig. 6B).

Analysis of The Cancer Genome Atlas (TCGA) database of human HNSCCs suggested a significant $(P<0.02)$ reduction in VPS4B mRNA levels in HNSCC cells that harbor increased miR-125b gene copy numbers (Fig. 6C). Additionally, $>50 \%$ of HNSCCs showed a loss of at least one VPS $4 B$ allele, a phenomenon not observed for closely related VPS4A or VDR (Fig. 6D). VPS4B homozygous deletions also correlated with poor survival rate (Fig. 6E), and reduced VPS $4 B$ mRNA levels correlated with elevated EGFR phosphorylation (Fig. 6F). Collectively, these data are consistent with the notion that miR-125b targets $V P S 4 B$, which has tumor suppressor activity in HNSCCs.

To determine whether $V p s 4 b$ down-regulation might be partially responsible for the positive effects of $\mathrm{miR}-125 \mathrm{~b}$ on ERK activity in DTG MKs, we transduced them with a Dox-inducible expression cassette of Vps $4 b$ cDNA that lacks its miR-125b-regulated 3' UTR. The resulting DTG/ $T R E-V p s 4 b$ MKs allowed us to simultaneously overexpress VPS4B protein and miR-125b upon Dox induction. As predicted, VPS4B overexpression counteracted the elevated ERK phosphorylation caused by miR-125b overexpression; it also suppressed the effects of miR-125b in permitting anchorage-independent protection against anoikis (Fig. 6G-I).

miR-125b-overexpressing DTG-CSCs could not be cultured in vitro (data not shown). Therefore, to assess the role of $V p s 4 b$ in miR-125b-addicted tumor growth in vivo, we cloned the TRE-Vps $4 b$ cassette into a lentiviral vector constitutively expressing H2BRFP and infected a suspension of $10^{6}$ FACS-isolated GFP ${ }^{+}$DTG-CSCs with either TRE-Vps $4 b$ or empty vector control virus. One hour later, cells were washed and intradermally engrafted into Nude mice fed on Dox food to initiate DTG tumor growth. As an infection rate control, $10^{6}$ uninduced DTG MKs were similarly infected and then plated in vitro. Subsequent FACS analysis revealed that DTG-CSCs transduced with the TRE-Vps $4 b$, but not vector control, were specifically depleted in the tumor, while in uninduced MK cultures, both $V p s 4 b$ and control were comparably represented (Fig. 6J; Supplemental Fig. S6A,B). These data suggest that overexpression of VPS4B can suppress growth of miR-125b-addicted DTG-CSCs in vivo.

\section{miR-125b regulates stress-responsive MAPK pathway} via multiple target genes

The function of a miRNA is often carried out by simultaneously fine-tuning multiple targets involved in a biological pathway. Using the Database for Annotation, Visualization, and Integrated Discovery (DAVID) (Dennis et al. 2003), we found that the MAPK signaling pathway was the only significantly enriched biological pathway among our 165 putative miR-125b targets in DTG-CSCs (Supplemental Fig. S6C). Closer inspection of putative miR-125b targets in this category revealed transcripts encoding stress-responsive MAPKs (Sugden and Clerk 
A

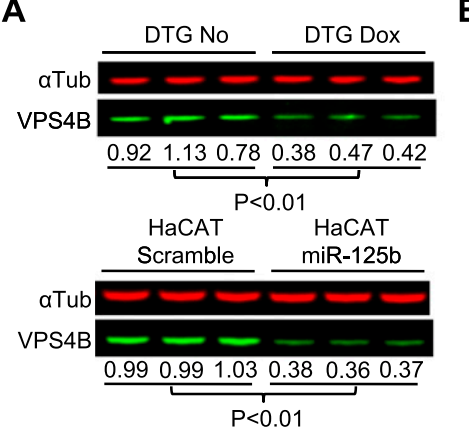

D

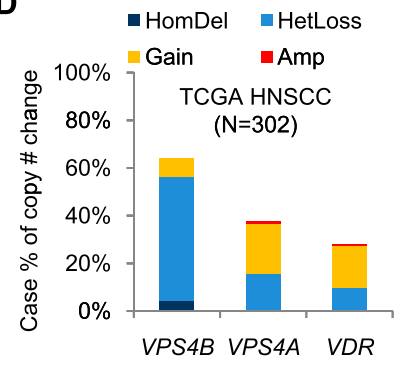

G

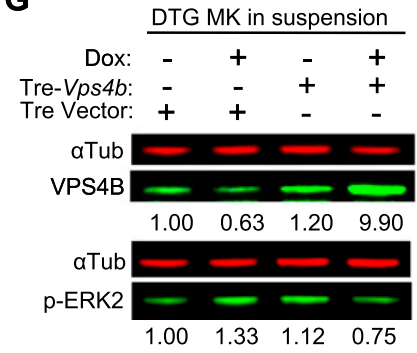

E
B

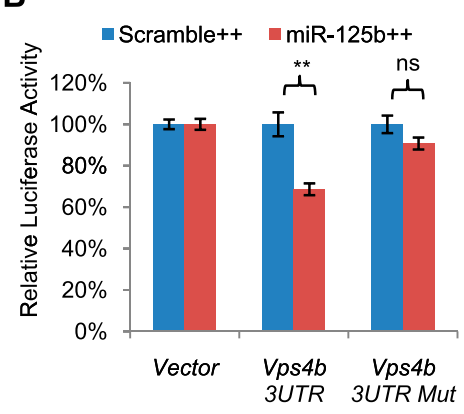

C

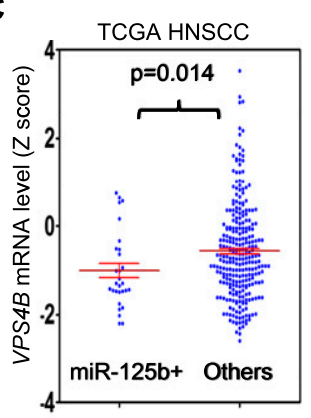

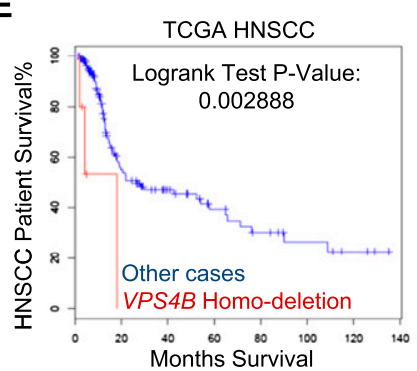

F TCGAHNSCC

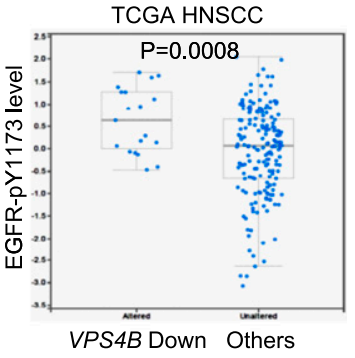

H DTG MK in suspension

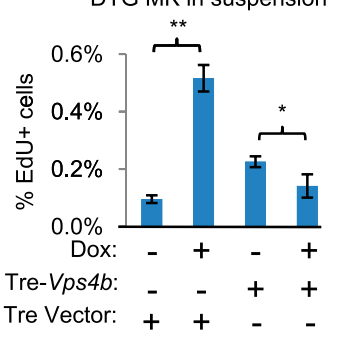

I

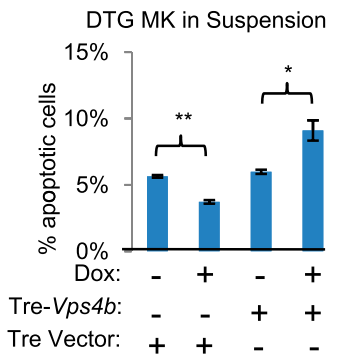

J

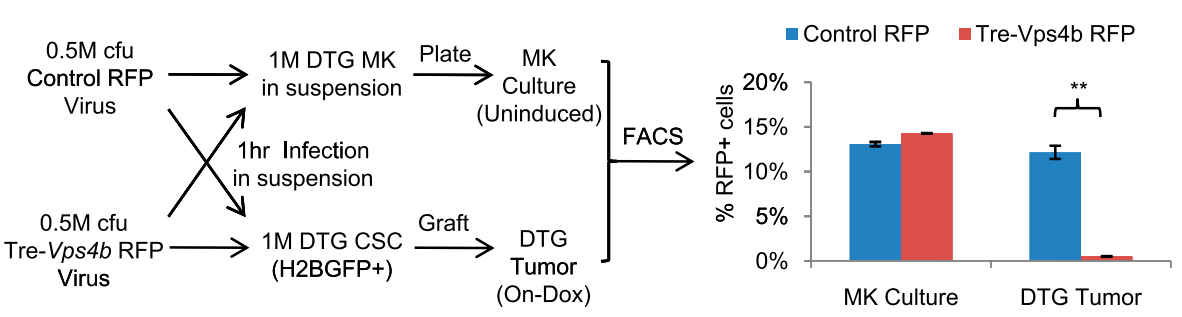

Figure 6. $V p s 4 b$ is a direct $m i R-125 b$ target in mice and humans. $(A)$ Two-color quantitative immunoblot analysis (each lane is an independent well) of off-Dox regressing DTG-CSCs (top panel), cultured DTG MK cells $\pm 24 \mathrm{~h}$ of Dox induction (middle panel), and HaCAT cells overexpressing miR-125b or scrambled control (bottom panel). All features are as described in Figure 5, B and C. (B) Dualluciferase reporter assay for a full-length mouse $V p s 4 b 3^{\prime}$ UTR and its miR-125b target site mutated version (Mut). Reporters were cotransfected into cultured wild-type (WT) MKs harboring Dox-inducible versions of either scrambled++ (control) or miR-125b++ (miR125b). $n=4$ independent wells. $\left.{ }^{\star *}\right) P<0.01$; (ns) not significant, two-tailed $t$-test. $(C)$ The level of VPS4B mRNA (Z-score) in samples of HNSCCs (TCGA) carrying an increased total miR-125b-1 miR-125b-2 gene copy number (miR-125b ${ }^{+}$or others. (Red lines) Mean value and standard error; (dot) individual HNSCC case. $P$-value is based on two-tail $t$-test. $(D)$ The percentage of HNSCCs (TCGA) bearing copy number changes in the indicated genes. (HomDel) Homozygous deletion; (HetLoss) heterozygous loss; (Gain) less than twofold copy number increase; (Amp) twofold or more copy number increase. (E) Patient survival curves of TCGA HNSCCs harboring VPS4B homozygous deletion (red) versus other cases (blue). Data were analyzed by the cBioPortal tool. $P$-value is based on two-tailed log rank test. (F) Levels of EGFR(Y1173) phosphorylation (TCGA RPPA score) in TCGA HNSCC samples (blue dots) with down-regulated VPS4B mRNA $(Z$-score $<-2)$ compared with other cases. Data were analyzed by the cBioPortal tool. $P$-value is based on two-tail $t$-test. $(G)$ Two-color quantitative immunoblot analysis of DTG MKs after $12 \mathrm{~h}$ of suspension and with indicated modifications. (Tre-Vps $4 b$ ) pLKO-Tre2-Vps $4 b$ cDNA lentivirus-infected; (Tre vector) pLKO-Tre2 lentivirus-infected. $(H, I)$ The percentage of apoptotic cells (AnnexinV [+] and dead cell stain [-]) and EdU ${ }^{+}$cells $(1 \mathrm{~h}$ after EdU labeling) in suspended DTG MKs with the indicated modifications. $n=3$ independent wells. $\left(^{\star}\right) P<0.05{ }^{*}\left(^{\star}\right) P<0.01$, two-tailed $t$-test. $(J)$ Illustration and quantification for the effect of $V p s 4 b$ overexpression on in vivo tumor growth of GFP+DTG-CSCs. MK cultures were FACS-analyzed $48 \mathrm{~h}$ post-infection $(n=3$ independent wells). DTG tumors were FACS-analyzed once the tumor reached $\sim 1 \mathrm{~cm}$ diameter $\left(n=3\right.$ independent tumors). $\left(^{\star \star}\right) P<0.01$, two-tailed $t$-test. 
Zhang et al.

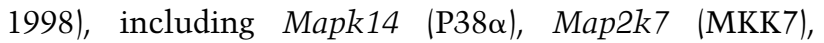
Map3k1 (MEKK1), and Map3k11 (MLK3) (Fig. 7A).

These MAPK mRNAs were significantly up-regulated concomitant with miR-125b down-regulation in DTGregressing tumors (Supplemental Table S1). Moreover, immunoblot analyses showed that DTG tumor regression was accompanied by potent phosphorylation (activation) of P38, SAPK/JNK, and JUN (Fig. 7B). Analysis of the TCGA revealed that an increased $m i R-125 b$ copy number in HNSCC patients was significantly associated
A
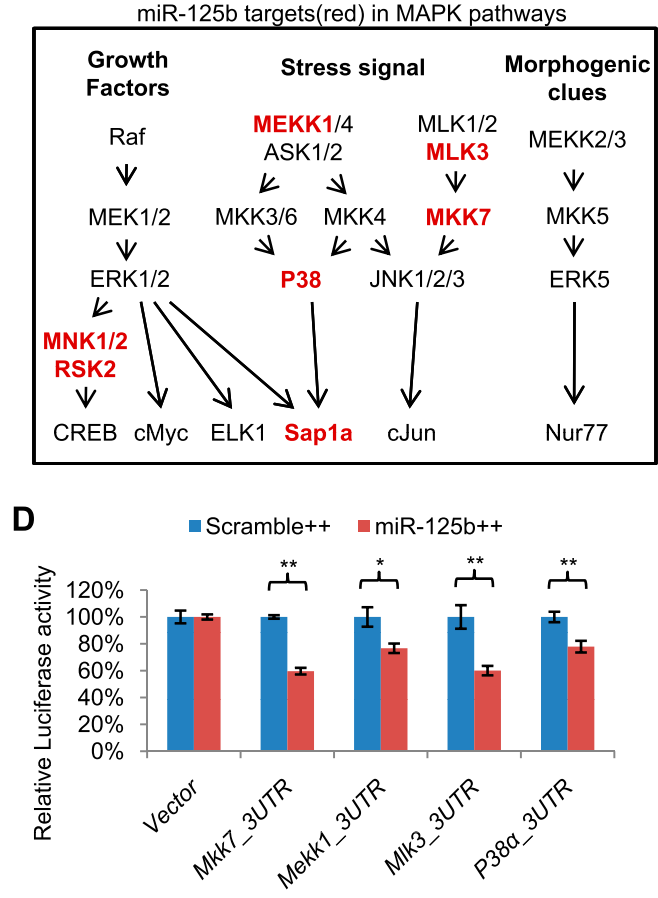

B

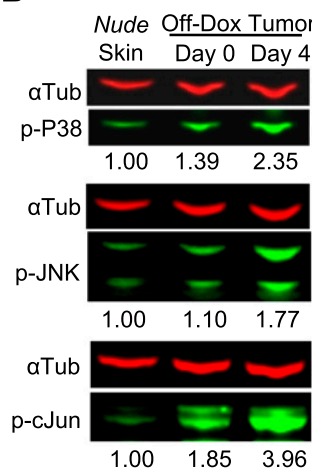

C

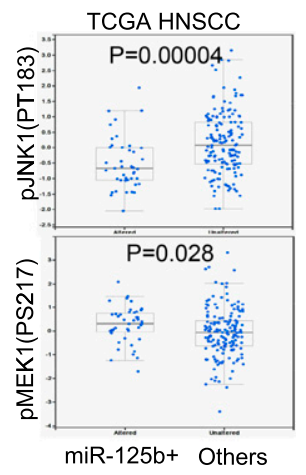

E

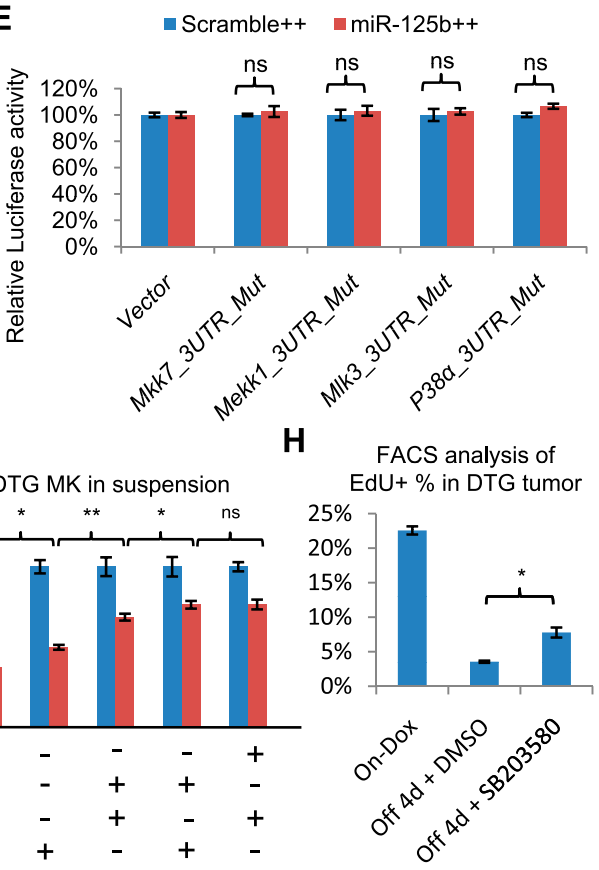

Figure 7. $m i R-125 b$ regulates MAPK signaling pathways via multiple direct targets. $(A)$ A simplified view of putative miR-125b targets (red) in MAPK signaling pathways. (B) Two-color quantitative immunoblot analysis of lysates from Nude skin-engrafted DTG tumors during off-Dox regression. Proteins of interest levels (green) were quantified relative to $\alpha$-Tubulin loading control (red) and compared with the value of Nude skin. (C) Levels of JNK1 (PT183) and MEK1 (PS217) phosphorylation (TCGA RPPA score) in HNSCC samples of patients with an increased miR-125b-1+miR-125b-2 gene copy number $\left(m i R-125 b^{+}\right)$versus others. $P$-value is based on unpaired twotailed $t$-test. $(D, E)$ Dual-luciferase reporter assay to test $m i R$-125b's regulation on stress-responsive MAPKs. Four putative direct targets-Mkk7, Mekk1, Mlk3, and $p 38 \alpha$-were tested in wild-type (WT) MKs \pm miR-125b overexpression. (D) Wild-type 3' UTRs. (E) 3' UTRs with miR-125b target site mutated (Mut). The vertical axis indicates relative luciferase activity. (Scrambled++) Scrambled control; (miR-125b++) miR-125b overexpression. $n=4$ independent wells. $\left({ }^{\star}\right) P<0.05 ;\left(^{\star \star}\right) P<0.01$; (ns) not significant, two-tail $t$-test. $(F)$ Two-color quantitative immunoblot analysis (each lane represents an independent well) of cultured DTG MK cells $24 \mathrm{~h}$ after Dox induction. Proteins of interest levels (green) were quantified relative to $\alpha$-Tubulin loading control (red) and compared with the average value of the first three left columns of each figure. $P$-value is based on two-tailed $t$-test. (G) MTT cell proliferation/survival assay of DTG MKs with the indicated modifications after 0 or $48 \mathrm{~h}$ in suspension. $V p s 4 b$ and control shRNAs were introduced by lentiviral infection and puromycin selection. $n=8$ independent wells. $\left.\left({ }^{\star}\right) P<0.05 ;{ }^{\star \star}\right) P<0.01$; (ns) not significant, two-tailed $t$-test. $(H)$ FACS analysis of DTG tumors after $4 \mathrm{~h}$ of EdU labeling. (On-Dox) DTG tumor on continuous Dox induction; (Off-Dox $4 \mathrm{~d}+\mathrm{DMSO}$ or SB203580) mice with DTG tumors that developed while on Dox were taken off Dox for $4 \mathrm{~d}$ and received daily injections of $10 \mu \mathrm{g}$ per gram of body weight of SB203580 or DMSO solvent during these $4 \mathrm{~d}$. 
with a reduction in the activity of stress-responsive MAPK and JNK1 but a modest increase in the activity of MEK1 downstream from EGFR-Ras signaling (Fig. 7C). In this regard, it was notable that Ink1-null mice exhibit increased susceptibility to DMBA/TPA-induced skin tumor formation (She et al. 2002), similar to the effects of $m i R-125 b$ overexpression.

TargetScan predicted conserved miR-125b-binding sites in the 3' UTR of all four stress-responsive MAPKS mentioned above (Supplemental Fig. S6D). To test their efficacy, we cloned the $\sim 500$-nucleotide (nt) 3' UTR regions encompassing each site into a dual-luciferase reporter system and then assayed their activities in cultured MKs. As expected for bona fide miR targets, all four reporters showed reduced activities upon miR-125b elevation. The effects were abolished when the seed regions of predicted miR-125b-binding sites were mutated (Fig. 7D,E). At the protein level, miR-125b overexpression significantly reduced both P38 and MEKK1 in MK culture (Fig. 7F). Moreover, in normal cultured (DTG) MKs, P38/JNK inhibitor SB203580 and Vps4b knockdown each improved survival in suspension culture, and their combined effect was comparable with Dox-induced miR-125b overexpression (Fig. 7G; Supplemental Fig. S6E,F). In vivo, SB203580 also partially suppressed the negative effects of miR-125b withdrawal on DTG tumor proliferation (Fig. $7 \mathrm{H}$ ).

Together, these data point to the view that $m i R-125 b$ 's effects on malignant transformation are mediated through a multiplicity of targets, including both $V p s 4 b$ and stress response Mapks. This is reminiscent of findings in Drosophila melanogaster, where dominant-negative mutants of $V p s 4 b(d V p s 4)$ and Jnk (Bsk) together but not individually led to neoplastic growth in imaginal disc epithelia (Rodahl et al. 2009).

\section{Discussion}

Our data provide compelling evidence that $m i R-125 b$ can enhance initiation and malignant progression of skin tumors formed spontaneously or by DMBA/TPA. Moreover, tumor-initiating cells derived from miR-125b-overexpressing tumors are addicted to this miRNA. These in vivo functional data unmask the oncogenic potential of elevated $m i R-125 b$ and, to our knowledge, provide the first example of oncomiR addiction for an epithelial tumor.

miR-125b overexpression also resulted in suprabasal proliferation and delayed differentiation in epidermis and increased survival in suspension cultures. From these results, it is tantalizing to speculate that miR-125b may be involved in developing anchorage independency during malignant progression. Consistent with this hypothesis, miR-125b levels are substantial at early but not late stages of mouse and human tumorigenesis. However, even for those human SCC cell lines that display downregulated $m i R-125 \mathrm{~b}$, overexpression of this $\mathrm{miR}$ improved their anchorage independency, while further knockdown of endogenous miR-125b repressed tumor growth in xenotransplantations. These results underscore the benefits of even low levels of miR-125b in SCC cells.
That said, deletions of the miR-125b locus can occur in advanced human SCCs. Whether miR-125b's loss is compensated by the simultaneous deletion and/or repression of tightly linked let-7 tumor suppressors remains to be determined.

How, then, does $m i R-125 b$ exert its influence on tumor promotion? Our data suggest a complexity that cannot be simply packaged in a linear pathway. First, by suppressing epidermal differentiation and promoting proliferation, $m i R-125 b$ overexpression is likely to precipitate a tumor-prone environment by perturbing the skin barrier. This could provoke a proinflammatory response, which would further fuel hyperproliferation. That said, miR$125 b$ 's effects on expression profiles of DTG-CSCs were profound. Thus, in our miR-125b addiction model, the progression from a normal skin stem cell transcriptional profile to an SCC-CSC-like one appeared to be at least partially reversible. Withdrawal of Dox in our miR$125 b$-addicted CSCs shifted their transcriptional profile toward one more similar to that of normal skin stem cells. This provides an interesting example in which a single miRNA can markedly impact the molecular identity of SCC-CSCs.

Finally, the myriad of miR-125b's potential direct targets in both skin stem cells and DTG-CSCs was also profound. Our studies here exposed miR-125b as a potential regulator of EGFR and MAPK signaling, which are key pathways in epithelial cancers. On the one hand, miR-125b indirectly boosted EGFR-Ras-MEK-ERK activities, which in part appeared to be by targeting the mRNA encoding VPS4B, which may interfere with recycling internalized active EGFR back to the epithelial cell's surface, as previously suggested (Lin et al. 2012). Although VPS4B is a generally important endocytosis protein whose function could go well beyond EGFR regulation, its effects on EGFR could account for many of miR-125b's effects observed here.

On the other hand, miR-125b directly targeted and dampened genes involved in stress-responsive MAPK cascades. In contrast to the EGFR pathway, the roles of stress-responsive MAPK pathways in cancer are complicated and can be either oncogenic or tumor-suppressive depending on cellular context (Wagner and Nebreda 2009). miR-125b's ability to directly target these Mapk mRNAs could contribute to the complex and often paradoxical roles of miR-125b in different types/stages of cancer.

In closing, it merits emphasizing that the effects of a miRNA often reflect cumulative effects of simultaneously targeting many protein-coding transcripts. Hence, it is likely that other miR-125b targets beyond $V p s 4 b$ and Mapks will contribute to the oncogenic effects that we described here. In this regard, it is interesting that in hematopoietic stem cells (HSCs), miR-125b was recently found to regulate multiple target genes involved in TGF- $\beta$ signaling (Emmrich et al. 2014).TGF- $\beta$ signaling also functions in skin tumorigenesis, and Smad4, one of the miR-125b targets in HSCs, was also among our miR-125b targets (Supplemental Table S1, sheet 1). Another previously reported miR-125b target is TACSTD2, which functions in MAPK signaling (Nakanishi et al. 2014). Even 
though TACSTD2 lacks a TargetScan-predicted miR-125bbinding site on its $3^{\prime}$ UTR, it negatively correlated with miR-125b in our DTG tumor regression data set (Supplemental Table S1, sheet 2). Another important factor in skin tumorigenesis is inflammation, and, in lymphomas, miR$125 b$ has a positive effect on the NF-кB pathway (Kim et al. 2012). We also detected significant enrichment of the NF$\kappa \mathrm{B}$ pathway as well as proinflammatory cytokines such as CCL2, CCL20, and CCL7 in our cohort of transcripts that positively correlated with miR-125b in our DTG tumor regression data set (Supplemental Table S1, sheet 3; data not shown). Overall, our data reconcile well with previous knowledge of $m i R-125 b$ targets and provide novel insights into the wide spectrum of biological functions regulated by $m i R-125 b$. In providing the first transcriptome analysis of miR-125b-mediated oncomiR addiction in vivo, our study also unveils a rich list of putative miR-125b targets with potential relevance to epithelial oncogenesis.

\section{Materials and methods}

\section{Animal care and use}

All animal experiments were performed in the Association for Assessment and Accreditation of Laboratory Animal Care (AAALAC)accredited Animal Resource Center at The Rockefeller University. Experiments were in accordance with National Institutes of Health guidelines for animal care and use and were approved and overseen by The Rockefeller University's Laboratory Animal Resource Center and animal care committee.

Tumor induction, tumor cell manipulation, and transplantations

DMBA/TPA tumor induction in age-matched female mice was according to standard protocols (Filler et al. 2007). For tumor cell transplantation experiments, cells were FACS-isolated, mixed with Matrigel (BD Biosciences), and then injected intradermally into Nude mice as described (Schober and Fuchs 2011). Serial intradermal transplantations were conducted with $1 \times 10^{4}$ freshly FACS-purified $\alpha 6^{\text {hi }} \beta 1^{\text {hi }}$ DTG tumor cells per site. For H2BGFP marking of DTG tumor-initiating cells, the PLKO-PGK-H2B-GFP vector (Beronja et al. 2010) without $U 6$ promoter was packaged into lentivirus and used to infect $\sim 1 \times 10^{6}$ freshly isolated $\alpha 6^{\text {hi }} \beta 1^{\text {hi }}$ DTG tumor cells. One hour after infection, cells were washed and engrafted directly onto backskins of Nude mice by intradermal transplantation. GFP $+\alpha 6^{\text {hi }} \beta 1^{\text {hi }}$ cells were FACS-isolated from resulting tumors and then serially transplanted as above.

Vitamin D ligand EB1089 administration was conducted as described (Zhang et al. 2011). Erlotinib (Santa Cruz Biotechnology) was resuspended in $0.5 \%(\mathrm{w} / \mathrm{v})$ methylcellulose in water and administered daily by intraperitoneal (i.p.) injection of $50 \mu \mathrm{g}$ per gram of body weight. An equal volume of solvent was injected as a negative control. SB203580 (Cell Signaling) was dissolved in DMSO and used at $10 \mu \mathrm{M}$ in vitro or i.p. injected into mice at 10 $\mu \mathrm{g}$ per gram of body weight daily. FACS isolation of SCC-CSCs and normal skin stem cells was conducted as described (Schober and Fuchs 2011).

\section{Pathology and human tissues}

Mouse tumors were analyzed by the Pathology Core Facility of the Memorial Sloan Kettering Cancer Center. Human skin cancer tissue arrays (SK801b, BC21014, and SK961) were purchased from Biomax.

Isolation of mRNAs from tumor-initiating cells of regressing miR-125b-addicted tumors

For RNA sequencing (RNA-seq) analysis of the miR-125baddicted tumor regression process, H2BGFP-labeled DTG tumor cells were engrafted intradermally into Nude mice $\left(1 \times 10^{4}\right.$ cells per site). Tumors were allowed to grow to $\sim 1 \mathrm{~cm}$ diameter on Dox. Mice were then taken off Dox by transferring them to regular food (Zhang et al. 2011) for the times noted in the text. For GFP $+\alpha 6^{\text {hi }} \beta 1^{\text {hi }}$ FACS isolations of tumor cells, two or three independent replicates were collected for each time point. Total RNAs from cells were extracted using the miRNeasy minikit (Qiagen). Expression of miR-125b in each sample was quantified by qPCR using TaqMan microRNA assays (Applied Biosystems).

\section{Data deposit}

The complete RNA-seq data were submitted to the NCBI Gene Expression Omnibus repository under accession number GSE54704.

\section{Acknowledgments}

We thank the Fuchs laboratory for helpful suggestions and shared reagents relating to this project. We also thank the Rockefeller University Comparative Biology Center for health care for our mice, the Pathology Core Facility of Memorial Sloan Kettering Cancer Center for pathology analysis of tumor samples, and Dr. Cynthia Magro at the Weill Cornell Medical College for normal human skin and AK samples. E.F. is a Howard Hughes Medical Institute (HHMI) Investigator. The work was supported by HHMI, a grant from the National Institutes of Health, and the Kimberly Lawrence-Netter Cancer Research Discovery Fund. L.Z. and E.F. designed experiments, analyzed data, and wrote the paper. Y.G. conducted the in situ experiments. L.Z. performed all other experiments, collected the data, and prepared the figures. E.F. supervised the study.

\section{References}

Abel EL, Angel JM, Kiguchi K, DiGiovanni J. 2009. Multi-stage chemical carcinogenesis in mouse skin: fundamentals and applications. Nat Protoc 4: 1350-1362.

Banzhaf-Strathmann J, Edbauer D. 2014. Good guy or bad guy: the opposing roles of microRNA $125 \mathrm{~b}$ in cancer. Cell Commun Signal 12: 30.

Barbie DA, Tamayo P, Boehm JS, Kim SY, Moody SE, Dunn IF, Schinzel AC, Sandy P, Meylan E, Scholl C, et al. 2009. Systematic RNA interference reveals that oncogenic KRAS-driven cancers require TBK1. Nature 462: 108-112.

Beronja S, Livshits G, Williams S, Fuchs E. 2010. Rapid functional dissection of genetic networks via tissue-specific transduction and RNAi in mouse embryos. Nat Med 16: 821-827.

Blanpain C, Lowry WE, Geoghegan A, Polak L, Fuchs E. 2004. Self-renewal, multipotency, and the existence of two cell populations within an epithelial stem cell niche. Cell 118: 635-648.

Bousquet M, Quelen C, Rosati R, Mansat-De Mas V, La Starza R, Bastard C, Lippert E, Talmant P, Lafage-Pochitaloff M, Leroux D, et al. 2008. Myeloid cell differentiation arrest by miR-125b-1 in myelodysplastic syndrome and acute myeloid leukemia with the $\mathrm{t}(2 ; 11)(\mathrm{p} 21 ; \mathrm{q} 23)$ translocation. I Exp Med 205: 2499-2506. 
Bousquet M, Harris MH, Zhou B, Lodish HF. 2010. MicroRNA miR125b causes leukemia. Proc Natl Acad Sci 107: 21558-21563.

Calin GA, Sevignani C, Dumitru CD, Hyslop T, Noch E, Yendamuri S, Shimizu M, Rattan S, Bullrich F, Negrini M, et al. 2004. Human microRNA genes are frequently located at fragile sites and genomic regions involved in cancers. Proc Natl Acad Sci 101: 2999-3004.

Chaudhuri AA, So AY, Mehta A, Minisandram A, Sinha N, Jonsson VD, Rao DS, O'Connell RM, Baltimore D. 2012. Oncomir miR-125b regulates hematopoiesis by targeting the gene Lin28A. Proc Natl Acad Sci 109: 4233-4238.

Cockerell CJ. 2000. Histopathology of incipient intraepidermal squamous cell carcinoma ('actinic keratosis'). I Am Acad Dermatol 42: 11-17.

Creighton CJ, Hilger AM, Murthy S, Rae JM, Chinnaiyan AM, El-Ashry D. 2006. Activation of mitogen-activated protein kinase in estrogen receptor $\alpha$-positive breast cancer cells in vitro induces an in vivo molecular phenotype of estrogen receptor $\alpha$-negative human breast tumors. Cancer Res 66: 3903-3911.

Dennis G Jr, Sherman BT, Hosack DA, Yang J, Gao W, Lane HC, Lempicki RA. 2003. DAVID: Database for Annotation, Visualization, and Integrated Discovery. Genome Biol 4: 3.

Driessens G, Beck B, Caauwe A, Simons BD, Blanpain C. 2012. Defining the mode of tumour growth by clonal analysis. Nature 488: 527-530.

Ebert MS, Neilson JR, Sharp PA. 2007. MicroRNA sponges: competitive inhibitors of small RNAs in mammalian cells. Nat Methods 4: 721-726.

Emmrich S, Rasche M, Schoning J, Reimer C, Keihani S, Maroz A, Xie Y, Li Z, Schambach A, Reinhardt D, et al. 2014. miR$99 \mathrm{a} / 100 \sim 125 \mathrm{~b}$ tricistrons regulate hematopoietic stem and progenitor cell homeostasis by shifting the balance between TGF $\beta$ and Wnt signaling. Genes Dev 28: 858-874.

Enomoto Y, Kitaura J, Hatakeyama K, Watanuki J, Akasaka T, Kato N, Shimanuki M, Nishimura K, Takahashi M, Taniwaki $\mathrm{M}$, et al. 2011. Emu/miR-125b transgenic mice develop lethal B-cell malignancies. Leukemia 25: 1849-1856.

Filler RB, Roberts SJ, Girardi M. 2007. Cutaneous two-stage chemical carcinogenesis. CSH Protoc doi: 10.1101/pdb. prot4837.

Friedman RC, Farh KK, Burge CB, Bartel DP. 2009. Most mammalian mRNAs are conserved targets of microRNAs. Genome Res 19: 92-105.

Guo S, Bai H, Megyola CM, Halene S, Krause DS, Scadden DT, Lu J. 2012. Complex oncogene dependence in microRNA125a-induced myeloproliferative neoplasms. Proc Natl Acad Sci 109: 16636-16641.

Hennings H, Shores R, Wenk ML, Spangler EF, Tarone R, Yuspa SH. 1983. Malignant conversion of mouse skin tumours is increased by tumour initiators and unaffected by tumour promoters. Nature 304: 67-69.

Henson BJ, Bhattacharjee S, O'Dee DM, Feingold E, Gollin SM. 2009. Decreased expression of miR-125b and miR-100 in oral cancer cells contributes to malignancy. Genes Chromosomes Cancer 48: 569-582.

Jin Z, Xu S, Yu H, Yang B, Zhao H, Zhao G. 2013. miR-125b inhibits Connexin 43 and promotes glioma growth. Cell Mol Neurobiol 33: 1143-1148.

Johnson SM, Grosshans H, Shingara J, Byrom M, Jarvis R, Cheng A, Labourier E, Reinert KL, Brown D, Slack FJ. 2005. RAS is regulated by the let-7 microRNA family. Cell 120: 635-647.

Kim SW, Ramasamy K, Bouamar H, Lin AP, Jiang D, Aguiar RC. 2012. MicroRNAs miR-125a and miR-125b constitutively activate the NF-кB pathway by targeting the tumor necrosis factor $\alpha$-induced protein 3 (TNFAIP3, A20). Proc Nat1 Acad Sci 109: 7865-7870.

Klusmann JH, Li Z, Bohmer K, Maroz A, Koch ML, Emmrich S, Godinho FJ, Orkin SH, Reinhardt D. 2010. miR-125b-2 is a potential oncomiR on human chromosome 21 in megakaryoblastic leukemia. Genes Dev 24: 478-490.

Lapouge G, Youssef KK, Vokaer B, Achouri Y, Michaux C, Sotiropoulou PA, Blanpain C. 2011. Identifying the cellular origin of squamous skin tumors. Proc Natl Acad Sci 108: 7431-7436.

Lapouge G, Beck B, Nassar D, Dubois C, Dekoninck S, Blanpain C. 2012. Skin squamous cell carcinoma propagating cells increase with tumour progression and invasiveness. EMBO J 31: 4563-4575.

Le MT, Teh C, Shyh-Chang N, Xie H, Zhou B, Korzh V, Lodish HF, Lim B. 2009. MicroRNA-125b is a novel negative regulator of p53. Genes Dev 23: 862-876.

Lin HH, Li X, Chen JL, Sun X, Cooper FN, Chen YR, Zhang W, Chung Y, Li A, Cheng CT, et al. 2012. Identification of an AAA ATPase VPS4B-dependent pathway that modulates epidermal growth factor receptor abundance and signaling during hypoxia. Mol Cell Biol 32: 1124-1138.

Liotta LA, Kohn E. 2004. Anoikis: cancer and the homeless cell. Nature 430: 973-974.

Malanchi I, Peinado H, Kassen D, Hussenet T, Metzger D, Chambon P, Huber M, Hohl D, Cano A, Birchmeier W, et al. 2008. Cutaneous cancer stem cell maintenance is dependent on $\beta$-catenin signalling. Nature 452: 650-653.

Mao JH, To MD, Perez-Losada J, Wu D, Del Rosario R, Balmain A. 2004. Mutually exclusive mutations of the Pten and ras pathways in skin tumor progression. Genes Dev 18: 18001805.

Mayr C, Hemann MT, Bartel DP. 2007. Disrupting the pairing between let-7 and Hmga2 enhances oncogenic transformation. Science 315: 1576-1579.

Mootha VK, Lindgren CM, Eriksson KF, Subramanian A, Sihag S, Lehar J, Puigserver P, Carlsson E, Ridderstrale M, Laurila E, et al. 2003. PGC-1 $\alpha$-responsive genes involved in oxidative phosphorylation are coordinately downregulated in human diabetes. Nat Genet 34: 267-273.

Nakanishi H, Taccioli C, Palatini J, Fernandez-Cymering C, Cui R, Kim T, Volinia S, Croce CM. 2014. Loss of miR-125b-1 contributes to head and neck cancer development by dysregulating TACSTD2 and MAPK pathway. Oncogene 33: 702-712.

Palmer HG, Anjos-Afonso F, Carmeliet G, Takeda H, Watt FM. 2008. The vitamin D receptor is a Wnt effector that controls hair follicle differentiation and specifies tumor type in adult epidermis. PLOS ONE 3: e1483.

Quintanilla M, Brown K, Ramsden M, Balmain A. 1986. Carcinogen-specific mutation and amplification of Ha-ras during mouse skin carcinogenesis. Nature 322: 78-80.

Raymond E, Faivre S, Armand JP. 2000. Epidermal growth factor receptor tyrosine kinase as a target for anticancer therapy. Drugs 60: 15-23.

Rodahl LM, Haglund K, Sem-Jacobsen C, Wendler F, Vincent JP, Lindmo K, Rusten TE, Stenmark H. 2009. Disruption of Vps4 and JNK function in Drosophila causes tumour growth. PLoS ONE 4: e4354.

Schober M, Fuchs E. 2011. Tumor-initiating stem cells of squamous cell carcinomas and their control by TGF- $\beta$ and integrin/focal adhesion kinase (FAK) signaling. Proc Natl Acad Sci 108: 10544-10549.

She QB, Chen N, Bode AM, Flavell RA, Dong Z. 2002. Deficiency of c-Jun- $\mathrm{NH}(2)$-terminal kinase-1 in mice enhances skin tumor development by 12-O-tetradecanoylphorbol-13acetate. Cancer Res 62: 1343-1348. 
Zhang et al.

Shi XB, Xue L, Ma AH, Tepper CG, Kung HJ, White RW. 2011. miR-125b promotes growth of prostate cancer xenograft tumor through targeting pro-apoptotic genes. Prostate 71: 538-549.

Subramanian A, Tamayo P, Mootha VK, Mukherjee S, Ebert BL, Gillette MA, Paulovich A, Pomeroy SL, Golub TR, Lander ES, et al. 2005. Gene set enrichment analysis: a knowledgebased approach for interpreting genome-wide expression profiles. Proc Nat1 Acad Sci 102: 15545-15550.

Sugden PH, Clerk A. 1998. 'Stress-responsive' mitogen-activated protein kinases (c-Jun $\mathrm{N}$-terminal kinases and p38 mitogenactivated protein kinases) in the myocardium. Circ Res 83: 345-352.

Sumazin P, Yang X, Chiu HS, Chung WJ, Iyer A, Llobet-Navas D, Rajbhandari P, Bansal M, Guarnieri P, Silva J, et al. 2011. An extensive microRNA-mediated network of RNA-RNA interactions regulates established oncogenic pathways in glioblastoma. Cell 147: 370-381.

Sun YM, Lin KY, Chen YQ. 2013. Diverse functions of miR-125 family in different cell contexts. J Hematol Oncol 6: 6.

Tang F, Zhang R, He Y, Zou M, Guo L, Xi T. 2012. MicroRNA$125 \mathrm{~b}$ induces metastasis by targeting STARD13 in MCF-7 and MDA-MB-231 breast cancer cells. PLOS ONE 7: e35435.

Tay Y, Kats L, Salmena L, Weiss D, Tan SM, Ala U, Karreth F, Poliseno L, Provero P, Di Cunto F, et al. 2011. Codingindependent regulation of the tumor suppressor PTEN by competing endogenous mRNAs. Cell 147: 344-357.

Wagner EF, Nebreda AR. 2009. Signal integration by JNK and p38 MAPK pathways in cancer development. Nat Rev Cancer 9: 537-549.

Weinstein IB. 2002. Cancer. Addiction to oncogenes-the Achilles heal of cancer. Science 297: 63-64.

White AC, Tran K, Khuu J, Dang C, Cui Y, Binder SW, Lowry WE. 2011. Defining the origins of Ras/p53-mediated squamous cell carcinoma. Proc Natl Acad Sci 108: 7425-7430.

Xu N, Zhang L, Meisgen F, Harada M, Heilborn J, Homey B, Grander D, Stahle M, Sonkoly E, Pivarcsi A. 2012. MicroRNA$125 \mathrm{~b}$ down-regulates matrix metallopeptidase 13 and inhibits cutaneous squamous cell carcinoma cell proliferation, migration, and invasion. J Biol Chem 287: 29899-29908.

Yu X, Cohen DM, Chen CS. 2012. miR-125b is an adhesionregulated microRNA that protects mesenchymal stem cells from anoikis. Stem Cells 30: 956-964.

Zhang L, Stokes N, Polak L, Fuchs E. 2011. Specific microRNAs are preferentially expressed by skin stem cells to balance self-renewal and early lineage commitment. Cell Stem Cell 8: 294-308. 


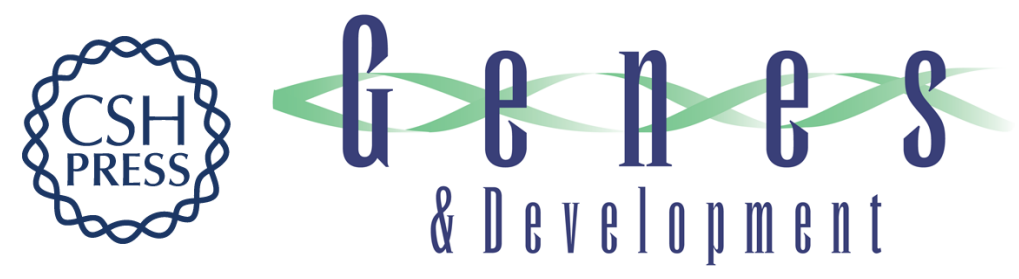

\section{miR-125b can enhance skin tumor initiation and promote malignant progression by repressing differentiation and prolonging cell survival}

Liang Zhang, Yejing Ge and Elaine Fuchs

Genes Dev. 2014, 28:

Access the most recent version at doi:10.1101/gad.248377.114

Supplemental http://genesdev.cshlp.org/content/suppl/2014/11/12/28.22.2532.DC1
Material

References This article cites 54 articles, 24 of which can be accessed free at: http://genesdev.cshlp.org/content/28/22/2532.full.html\#ref-list-1

Creative This article is distributed exclusively by Cold Spring Harbor Laboratory Press for the first Commons six months after the full-issue publication date (see

License http://genesdev.cshlp.org/site/misc/terms.xhtml). After six months, it is available under a Creative Commons License (Attribution-NonCommercial 4.0 International), as described at http://creativecommons.org/licenses/by-nc/4.0/.

Email Alerting Receive free email alerts when new articles cite this article - sign up in the box at the top Service right corner of the article or click here.

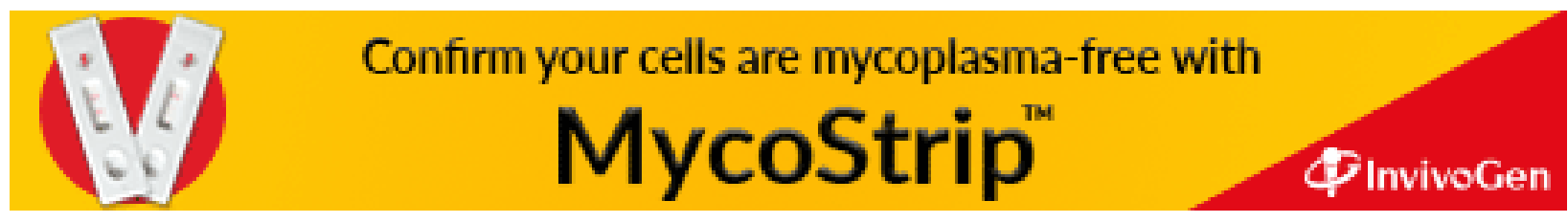

